\title{
Abaqus Implementation of Extended Finite Element Method
}

\author{
Using a Level Set Representation
}

\section{for Three-Dimensional Fatigue Crack Growth and Life Predictions}

\author{
Jianxu Shi ${ }^{1}$, David Chopp ${ }^{2}$, Jim Lua ${ }^{1}$, N. Sukumar ${ }^{3}$, and Ted Belytschko ${ }^{4}$ \\ ${ }^{1}$ Global Engineering and Materials, Inc. \\ One Airport Place, Princeton, NJ 08540 U.S.A. \\ ${ }^{2}$ Northwestern University, Dept. of Engineering Sciences and Applied Mathematics \\ 2145 Sheridan Road, Room M448, Evanston, IL 60208 U.S.A. \\ ${ }^{3}$ University of California at Davis, Dept. of Civil \& Environmental Engineering \\ One Shields Avenue, Davis, CA 95616 U.S.A. \\ ${ }^{4}$ Northwestern University, Dept. of Mechanical Engineering \\ 2145 Sheridan Road, Room A212, Evanston, IL 60208 U.S.A.
}

\begin{abstract}
A three-dimensional extended finite element method (X-FEM) coupled with a narrow band fast marching method (FMM) is developed and implemented in the Abaqus finite element package for curvilinear fatigue crack growth and life prediction analysis of metallic structures. Given the level set representation of arbitrary crack geometry, the narrow band FMM provides an efficient way to update the level set values of its evolving crack front. In order to capture the plasticity induced crack closure effect, an element partition and state recovery algorithm for dynamically allocated Gauss points is adopted for efficient integration of historical state variables in the near-tip plastic zone. An element-based penalty approach is also developed to model crack closure and friction. The proposed technique allows arbitrary insertion of initial cracks, independent of a base 3D model, and allows non-self-similar crack growth pattern without conforming to the existing mesh or local remeshing. Several validation examples are presented to demonstrate the extraction of accurate stress intensity factors for both static and growing cracks. Fatigue life prediction of a flawed helicopter lift frame under the ASTERIX spectrum load is presented to demonstrate the analysis procedure and capabilities of the method.
\end{abstract}

Keywords: X-FEM, stress intensity factor, crack growth, fatigue life prediction, fracture mechanics

\section{Nomenclature}

$\Delta a_{i}, \Delta a_{\max } \quad$ crack growth vector at tip $i$, maximum crack growth vector

$b_{i} \quad$ jump function displacement variables at node $i$ 
$B_{i, \text { con /jump /tip }}$

$B_{i}^{S}$

$c_{j i}$

C

$D^{s}$

D

E

$f$

$h_{c r}$

$H(\xi, \eta, \zeta)$

$\boldsymbol{F}^{\boldsymbol{s}}$

$F_{\text {el }}^{\text {ext }}$

$K_{\mathrm{I}}, K_{\mathrm{II}}, K_{\mathrm{III}}$

$K_{\text {eqv }}$

$\Delta K_{i}, \Delta K_{\max }$

$\Delta K_{\text {th }}$

$\Delta K_{\text {walker }}$

$\boldsymbol{K}_{\text {el }}$

$\boldsymbol{K}^{\mathrm{s}}$

m

$N_{i}(\xi, \eta, \zeta)$

$\Delta N$

$p$

$P_{1}, P_{2}, P^{*}$

P

$R$

$\boldsymbol{R}$

$\boldsymbol{R}_{\text {el }}$

$\mathrm{s}_{h}, \mathrm{~s}_{i}, s^{*}$

$S$

$T_{1}, T_{2}, T^{*}$

$T_{i}^{*}, T_{\max }^{*}$

$U_{i}$

$U_{\text {I }}, U_{\text {II }}, U_{\text {III }}$

$\boldsymbol{U}_{\boldsymbol{e l}}$

Greek Symbols

$\gamma$ strain-displacement matrix with respect to continuous/jump/tip displacements

global-local matrix to compute crack surface displacement jumps

displacement variables with respect to branch function $j$ at node $i$

fatigue parameter in the Paris law

tangent matrix in crack surface contact and frictional sliding

tangent matrix in material deformation

material Young's modulus

body force in a continuum body

critical value of crack surface penetration displacement

jump enrichment as function of reference coordinates $\xi, \eta, \zeta$

nodal reaction force in crack surface contact and friction

equivalent nodal external forces of the element

stress intensity factor in mode I, mode II, and mode III

equivalent stress intensity factor to compute crack growth vector

magnitude of $K_{\text {eqv }}$ within a load cycle at tip $i$, its maximum value

threshold value of $\Delta K$ in fatigue crack growth

modified $\Delta K$ in Walker's equation for fatigue crack growth

stiffness matrix of the element

penalty stiffness matrix in contact and frictional sliding

fatigue parameter in the Paris law

tri-linear shape function at node $i$

accumulated load cycles during an analysis increment

order of the polynomial used in state variable fitting

cut points, midpoint between $\psi=-r \cap \varphi=0$ and element edges

polynomial function used in state variable fitting

fatigue load ratio parameter

transformation matrix between global-local coordinates

nodal residuals of the element

state variable at Gauss point, element node $i$, and arbitrary location

crack surface area

cut points, midpoint between $\psi=0 \cap \varphi=0$ and element edges

tip point corresponding to $\Delta K_{i}$ and $\Delta K_{\max }$

displacement variables at node $i$

local displacement jumps in mode I, mode II, mode III components

nodal variables of the element

parameter in Walker's equation for fatigue crack growth 


\begin{tabular}{ll}
$\varepsilon_{i j}$ & material strain tensor \\
$(\xi, \eta, \zeta)$ & element reference coordinates \\
$\theta$ & angular variable in branch functions \\
$\kappa$ & material parameter in plain strain/plain stress calculations \\
$\mu$ & material parameter in plain strain/plain stress calculations \\
$v$ & material Possion's ratio \\
$\pi_{\mathrm{INT}}$ & virtual energy associated with crack surface interactions \\
$\sigma_{i j}$ & material stress tensor \\
$\tau$ & crack surface interaction force \\
$\varphi$ & level set variable describing signed distance to crack surface \\
$\psi$ & level set variable describing signed distance to crack front \\
$\Psi_{j}(r, \theta)$ & $\psi$ value at node $j$ with local polar coordinates $(r, \theta)$ \\
$\Omega, \partial \Omega$ & domain, boundary of domain of a continuum body \\
\hline
\end{tabular}

\section{Introduction}

Fracture and failure is particularly significant in the damage tolerance assessment of advanced metallic joints, since manufacturing flaws and in-service damage most often manifest themselves as initial cracks. To assess the criticality of an initial flaw and its impact on the residual strength and life, a finite element analysis is typically performed for various crack shapes, sizes, and locations. The finite element method poses certain limitations in such analyses since changes in the topology of the crack require remeshing of the domain. This tends to be a severe restriction and is burdensome for crack growth simulations in complex geometries. To alleviate the computational burden associated with the insertion of arbitrary cracks into an finite element model, the extended finite element method (X-FEM) (Belytschko and Black, 1999; Moes et al., 1999) has provided significant advantages over other approaches such as boundary element methods (Cruse, 1988), remeshing methods (Carter et al., 2000; Maligno et al., 2010), and element deletion methods (Henshell and Shaw, 1975). While the application of the boundary element method can accurately capture the near tip singularities, its extension to elasto-plastic fracture problems is quite awkward due to the use of a domain integration of fictitious body forces to account for the nonlinearity. An element deletion method is easy to implement, but it suffers from a severe dependence of the solution on the size and structure of the mesh. An automatic adaptive remeshing scheme can be difficult to apply to geometrically nonlinear problems involved in contact and friction of a growing crack, because of the large computational burden. The presence of multiple cracks will make the current state-of-the-art remeshing scheme intractable. In X-FEM, the finite element space is enriched with a discontinuous (jump) function and the near-tip asymptotic functions, which are added to the standard finite element approximation through the framework of partition of unity (Melenk and Babusk, 1996). Reviews on X-FEM are available in the literature (Karihaloo and Xiao, 2003; Abdelaziz, 2008; Belytschko et al., 2009). Numerous illustrations have demonstrated the key advantage of X-FEM, especially the ability to characterize arbitrarily shaped cracks in finite element methods without remeshing. 
With X-FEM, it is not necessary to reconstruct the finite element mesh as the problem evolves, therefore completely eliminating the need for remeshing.

Given the many attractive features of X-FEM in performing the damage tolerance assessment and simulating a curvilinear crack growth path in a complicated 3D geometry, several attempts have been promoted to integrate X-FEM with existing commercial FEM solvers. Sukumar et al. (2003) implemented X-FEM in Dynaflow (Prevost, 1983). Nisto et al. (2005) described implementation with an explicit dynamic code DynELA (Pantale et al., 2004); Bordas et al. (2006) integrated a 3D X-FEM coupled with level set method into a commercial FEM package I-DEAS; and very recently, Giner et al. (2009) implemented a 2D X-FEM within Abaqus. Indeed, another 2D X-FEM was implemented as an add-on library kit for Abaqus (Shi et al., 2008) that works seamlessly with the commercial, off-the-shelf (COTS) version of Abaqus/Standard and Abaqus/CAE for automated crack onset and growth. This 2D version was applied to fatigue life prediction considering residual stresses in Lua et al. (2008) and composite joints reliability assessment in Lua et al. (2009).

Driven by emerging needs in accurate and efficient characterization a surface crack in a complicated 3D geometry, the existing toolkit has to be extended for the 3D cases. The X-FEM capability was recently included in Abaqus Version 6.9, but only for a static crack. For a growing crack, a ghost node concept is employed, instead of nodal degree-of-freedom (DOF) enrichment with discontinuous functions, and the crack growth is driven by de-cohesion laws, instead of fracture mechanics criteria. Since the current design and certification of metallic structures conducted by major aerospace and ship industries are still based on the theory of linear elastic fracture mechanics (LEFM), an accurate calculation of the stress intensity factors in a growing crack is essential for performing the structural integrity and durability assessment. To meet such technical requirements and comply with the current damage tolerance design practices and certification guidelines, a discrete fracture mechanics approach has to be employed for the development of the 3D X-FEM toolkit for a commercial FEM solver.

The 3D X-FEM toolkit for Abaqus (XFA3D) has been developed and validated using a suite of benchmark problems. The main features of XFA3D include: 1) model preparation and arbitrary insertion of initial cracks that are independent of the base model via Abaqus/CAE; 2) extraction of stress intensity factors on static or growing crack fronts in metallic structures via the crack tip opening displacement (CTOD) and life predictions for constant or variable fatigue load; 3) accurate extraction of Strain Energy Release Rate (SERR) via the modified Virtual Crack Closure Technique (mVCCT) for composite applications; and 4) post-processing of the cracked region using Abaqus/Viewer. The critical components of XFA3D include: the level set representation and updates for a stationary and growing crack, the penalty approach for interface interactions in crack closure and friction, the element slicing state recovery algorithm for elasto-plastic fracture problems, and the extraction of fracture driving force along the crack front. A summary of the novel developments in these key solution modules of XFA3D is given below.

In XFA3D, a level set approach along with an enhanced fast marching method is integrated with 
$\mathrm{X}-\mathrm{FEM}$ to describe the initial crack geometry and track its growth. Stolarska et al. (2001) introduced the level set method (Osher and Sethian, 1988) into 2D X-FEM to describe a crack path. The 3D X-FEM coupled with the level set method was used to simulate non-planar crack growth in Moes et al. (2002) and Gravouil et al. (2002). In this approach a 3D crack is defined by two almost-orthogonal level sets that are derived from the signed distance functions. One function describes the crack surface in 3D space and the other is used to describe the crack front. With this implicit description of the crack front, an arbitrary evolving crack can be captured without explicit geometric representation of the crack. The 3D X-FEM was then coupled with the Fast Marching Method (Sethian, 1999), a more efficient level set algorithm, to solve single (Sukumar et al., 2003), multiple planar cracks (Chopp et al., 2003), or nonplanar crack growth (Sukumar et al., 2008). In this paper we further improve the level set update algorithm by introducing a narrow band concept for efficient update of the level sets for grid points near the front, a $\mathrm{C}^{(2)}$-smooth triquintic interpolation of level sets (Chopp, 2001), and a skin of the X-FEM region to identify the active level set zone for edge crack problems (with an opened crack front curve). These enhancements enable a more robust front tracking in the complicated 3D geometry where crack front turning, branching or elimination is inevitable during simulations.

In order to enhance the robustness and practicability for solving real industrial problems, the nonlinear material behavior especially in the vicinity of the near tip zone has to be considered. In plastic fracture mechanics, Elguedj et al. (2006) utilized the Hutchinson-Rice-Rosengren (HRR) fields containing six enrichment functions and a fixed distribution of Gauss points to obtain accurate prediction of near-tip plastic field for 2D problems. This approach in 3D would require 21 nodal DOFs and a large number of Gauss points per element. In this paper, a slicing scheme proposed by Sukumar et $a l$. (2000) is extended to 3D partially cut elements. If the crack propagates in a new direction, the element slicing scheme needs to be updated to be coherent with the new crack surface; therefore the locations of Guass points will change from the previous increment. In our implementation, the superconvergent patch recovery (Zienkiewicz et al., 1992), or SPR mapping is developed to recover the material states for the new set of Gauss points. Note that the state mapping is only needed for the new partially cut elements and when the plastic strains are not negligible. In practice, only a confined region (of several millimeters) around an active growing tip needs this state recovery step to account for the effects from the plastic zone. In other regions only minimal Gauss points are deployed as long as there are enough Gauss points to avoid weak singularity in the global stiffness matrix. For the current plastic fracture problems under small scale yielding we still use the four branch functions as in X-FEM formulation in LEFM. In fact, if calculation of displacement jumps in CTOD already takes into account plastic deformations, the $\mathrm{K}$ estimates are still accurate for a confined plasticity zone ahead of tips. To consider the effects of crack closure, frictional contact, or other interfacial forces, in this paper we also develop an element-based penalty contact approach following the principle of virtual work. Alternative approaches have been used, including the LATIN iterative scheme with 2D X-FEM as in Dolbow et al. (2001) the penalty contact with 2D X-FEM as in Khoei et al. (2006) and the Lagrange contact as in Khoei et al. (2009). We opt for a direct contact formulation to avoid new unknowns at the local level. Since a Lagrange approach cannot be easily implemented as a user element algorithm in Abaqus, the penalty approach appears to be a more rational choice for our implementation. 
The user subroutine interface, scripting-based CAE/GUI customization, and C++ ODB API available in the Abaqus package are utilized to create a complete toolkit for general fracture and fatigue analyses. In particular, the user element library (UEL) is used to implement X-FEM elements; the user external database (UEXTERNALDB) is used to control the analysis flow, to integrate an embedded FMM solver, and to store analysis data. In the presence of residual stresses, the initial stress subroutine (SIGINI) is used to introduce an initial stress condition. Isotropic elastic, elasto-plastic, and orthotropic elastic material models are implemented as the Abaqus user materials (UMAT) to be linked with the UEL. Customer material models can be linked using a very similar UMAT interface provided within the toolkit. Although only the linear, brick element type is fully implemented, its extension to other materials or elements can be easily achieved since the framework codes are written in $\mathrm{C}++$ with modularization. A basic GUI panel is developed to assist users to prepare X-FEM input files within Abaqus/CAE. At the end of load increments, analysis results are saved into a separate ODB file using the Abaqus C++ API, so that the users can use Abaqus/Viewer for post-processing needs. Parallel runs and the 64-bit platform (in Linux) are supported to provide access for larger applications. To our best knowledge, both the stress intensity factor prediction along a growing crack front and the fatigue life prediction are not available in the existing package of X-FEM in Abaqus Version 6.9.

The paper is organized as follows. A 3D X-FEM element formulation using a B-bar method is given in Section 2. Sections $3 \& 4$ describe an implicit representation of crack geometry, a triquintic interpolation of level sets, and a narrow band FMM for level set updates. In Section 5, a 3D crack front tracking and fatigue growth control algorithm is introduced. A penalty approach to characterize crack surface interactions, in particular contact and friction, is given in Section 6. Section 7 presents a slicing scheme to consider both completely cut and partially cut cases. In Section 8, an SPR state mapping algorithm is described for dynamically allocated material point integration. Section 9 provides a suite of example problems for parametric study and accuracy demonstration at sub-component level under monotonic and cyclic loading. Finally, in Section 10, an industrial example is presented to illustrate crack growth simulation and life prediction in a complex helicopter component under spectrum loading.

\section{A Modified 3D X-FEM Element Formulation to Avoid Mesh Locking}

In this section the 3D X-FEM is developed for both linear and nonlinear materials. In the standard $\mathrm{X}$-FEM formulation the displacements are approximated by

$$
\begin{aligned}
u(\xi, \eta, \zeta)= & \sum_{i} N_{i}(\xi, \eta, \zeta) U_{i}+\sum_{i} N_{i}(\xi, \eta, \zeta) H(\xi, \eta, \zeta) b_{i}+ \\
& \sum_{i} N_{i}(\xi, \eta, \zeta)\left(\sum_{j} \Psi_{j}(r, \theta) c_{j i}\right)
\end{aligned}
$$

where $N_{i}(\xi, \eta, \zeta)$ are shape functions; $U_{i} \in \mathbf{R}^{3}$ are nodal displacement parameters for all nodes of the hexahedral element: $1 \sim 8 ; b_{i} \in \mathbf{R}^{3}$ are jump function parameters at the jump-enriched nodes; and the $c_{j i} \in \mathbf{R}^{3} \times \mathbf{R}^{4}$ are branch function parameters at the tip-enriched nodes. The jump function $H$ is 
defined as the sign of level set $\varphi$ :

$$
H(\xi, \eta, \zeta)=\left\{\begin{array}{l}
+1: \varphi(\xi, \eta, \zeta)>0 \\
-1: \varphi(\xi, \eta, \zeta)<0 \\
\pm 1: \varphi(\xi, \eta, \zeta)=0
\end{array}\right.
$$

Note the function $H(\xi, \eta, \zeta)$ when $\varphi(\xi, \eta, \zeta)=0$ is not well defined; $H(\xi, \eta, \zeta)= \pm 1$ and $\llbracket H(\xi, \eta, \zeta) \rrbracket=2$ is only a convenient designation to evaluate the displacement jumps for a point sitting on the crack surface. The branch functions, noted $\Psi_{j}$, are also defined in a usual way by:

$$
\left\{\sqrt{r} \sin \left(\frac{\theta}{2}\right), \sqrt{r} \cos \left(\frac{\theta}{2}\right), \sqrt{r} \sin \left(\frac{\theta}{2}\right) \sin (\theta), \sqrt{r} \cos \left(\frac{\theta}{2}\right) \sin (\theta)\right\}
$$

where $r=\sqrt{\varphi^{2}(\xi, \eta, \zeta)+\psi^{2}(\xi, \eta, \zeta)}$ and $\theta=\operatorname{atan}\left(\frac{\varphi(\xi, \eta, \zeta)}{\psi(\xi, \eta, \zeta)}\right)$. The level set fields $\phi=\{\varphi, \psi\}$ in the X-FEM domain are interpolated by the shape functions:

$$
\phi(\xi, \eta, \zeta)=\sum_{i} N_{i}(\xi, \eta, \zeta) \Phi_{i} .
$$

In our 3D X-FEM elements the $B$ matrix is modified from the standard formulation to be fully compatible with Abaqus solid elements. The modified $B$ matrix is also known as the $B$-bar method (Nagtegaal et al., 1974), since the actual volume changes at the Gauss points are replaced by the average of volume change over the entire element. The modified strain-displacement relation helps to prevent mesh locking and therefore provides an accurate solution for highly incompressible cases. Using the modified $B$-matrix also ensures the derived X-FEM, if not cut by a crack, is fully compatible with the linear, fully integrated element in Abaqus.

To derive the strain operator we recall the deformation gradient: $\frac{\partial \Delta u}{\partial x}=\sum_{i} \frac{\partial N_{i}}{\partial \xi} \frac{\partial \xi}{\partial x} \Delta U_{i}=\sum_{i} N_{i, \xi} J^{-1} \Delta U_{i}$, where $J^{-1}$ is the inverse of the transformation matrix $J$. In the $B$-bar method we estimate the volumetric average of the terms in the deformation gradient operator, or $\left.\bar{N}_{i, \xi} \triangleq \frac{1}{V_{e}} \int_{V_{e}} N_{i, \xi} d v \approx N_{i, \xi}\right|_{\xi=0}$. If one denotes:

$$
\widetilde{\bar{N}}_{i, \xi}=\left(\left.N_{i, \xi}\right|_{\xi=0}-N_{i, \xi}\right) / 3,
$$

then the modified $B$-matrix for the unenriched nodes takes the form:

$$
B_{i, \text { con }}=J^{-1}\left[\begin{array}{ccc}
N_{i, \xi}+\widetilde{N}_{i, \xi} & \widetilde{N}_{i, \eta} & \widetilde{N}_{i, \zeta} \\
\widetilde{N}_{i, \zeta} & N_{i, \eta}+\widetilde{N}_{i, \eta} & \widetilde{\widetilde{N}}_{i, \zeta} \\
\widetilde{N}_{i, \zeta} & \widetilde{N}_{i, \eta} & N_{i, \zeta}+\widetilde{N}_{i, \zeta} \\
N_{i, \eta} & N_{i, \zeta} & 0 \\
N_{i, \zeta} & 0 & N_{i, \zeta} \\
0 & N_{i, \zeta} & N_{i, \eta}
\end{array}\right]
$$

where the derivatives of the shape functions are readily available. 
For the jump-enriched nodes the extra terms of the $B$-matrix are simply $B_{i, \text { jump }}=H B_{i, \text { con }}$, because the derivative of $H$ is zero. Note the jump function, $H$, is multiplied term by term in forming $B_{i, \text { con }}$, so the $B$-matrix is:

$$
B_{J}=\left[B_{J, \text { con }} \mid B_{J, \text { jump }}\right]
$$

where the subscript $J$ indicates the nodes with jump enrichment.

For the tip enriched nodes the extra terms of the $B$-matrix are:

$$
\begin{aligned}
& B_{i, j, \mathrm{tip}}=J^{-1}
\end{aligned}
$$

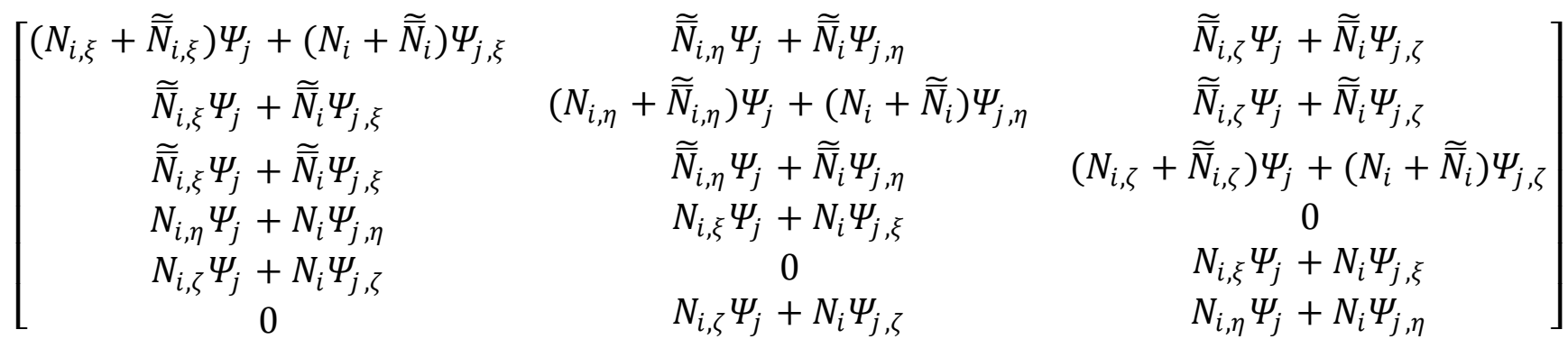

where the subscript, $j=1 \sim 4$, represent the branch functions. To obtain the derivatives of the branch functions, the chain rule is used, or $\Psi_{j, \xi}=\Psi_{j, r} r_{, \xi}+\Psi_{j, \theta} \theta_{, \xi}$, with $r_{, \xi}=r_{, \varphi} \varphi_{, \xi}+r_{, \psi} \psi_{, \xi}$ and $\theta_{, \xi}=$ $\theta_{, \varphi} \varphi_{, \xi}+\theta_{, \psi} \psi_{, \xi}$. The derivatives of the branch functions $\Psi_{j, r}$ and $\Psi_{j, \theta}$ are listed below:

$$
\begin{array}{ll}
\Psi_{1, r}=\frac{1}{2 \sqrt{r}} \sin \left(\frac{\theta}{2}\right) ; & \Psi_{1, \theta}=\frac{\sqrt{r}}{2} \cos \left(\frac{\theta}{2}\right) \\
\Psi_{2, r}=\frac{1}{2 \sqrt{r}} \cos \left(\frac{\theta}{2}\right) ; & \Psi_{2, \theta}=-\frac{\sqrt{r}}{2} \sin \left(\frac{\theta}{2}\right) \\
\Psi_{3, r}=\frac{1}{2 \sqrt{r}} \sin \left(\frac{\theta}{2}\right) \sin (\theta) ; & \Psi_{3, \theta}=\frac{\sqrt{r}}{2} \cos \left(\frac{\theta}{2}\right) \sin (\theta)+\sqrt{r} \sin \left(\frac{\theta}{2}\right) \cos (\theta) \\
\Psi_{4, r}=\frac{1}{2 \sqrt{r}} \cos \left(\frac{\theta}{2}\right) \sin (\theta) ; & \Psi_{4, \theta}=-\frac{\sqrt{r}}{2} \sin \left(\frac{\theta}{2}\right) \sin (\theta)+\sqrt{r} \cos \left(\frac{\theta}{2}\right) \cos (\theta)
\end{array}
$$

The transformation derivatives from $(\varphi, \psi)$ to $(r, \theta)$ are:

$$
r_{, \varphi}=\frac{\varphi}{r}, \quad r_{, \psi}=\frac{\psi}{r}, \theta_{, \varphi}=\frac{\psi}{r^{2}} \text {, and } \theta_{, \psi}=-\frac{\varphi}{r^{2}} .
$$

From Eqn (4), the level set derivatives $\varphi_{, \xi}$ and $\psi_{, \xi}$ can be interpolated in terms of shape function derivatives, namely:

$$
\varphi_{, \xi}=\sum_{i} N_{i, \xi} \Phi_{i}, \varphi_{, \eta}=\sum_{i} N_{i, \eta} \Phi_{i}, \varphi_{, \zeta}=\sum_{i} N_{i, \zeta} \Phi_{i} \text {, and }
$$




$$
\psi_{, \xi}=\sum_{i} N_{i, \xi} \Psi_{i}, \psi_{, \eta}=\sum_{i} N_{i, \eta} \Psi_{i}, \psi_{, \zeta}=\sum_{i} N_{i, \zeta} \Psi_{i}
$$

Finally the $B$-matrix for tip-enriched nodes can be written as:

$$
B_{T}=\left[B_{T, \text { con }}\left|B_{T, 1, \text { tip }}\right| B_{T, 2, \text { tip }}\left|B_{T, 3, \text { tip }}\right| B_{T, 4, \text { tip }}\right]
$$

where $T$ denotes the nodes with tip enrichment. The strain vector defined as $\boldsymbol{\varepsilon} \triangleq\left\{\varepsilon_{11}, \varepsilon_{22}, \varepsilon_{33}, \varepsilon_{12}, \varepsilon_{13}, \varepsilon_{23}\right\}$ is evaluated by:

$$
\boldsymbol{\varepsilon}=\sum_{k=1,8} B_{k} U_{k}
$$

where $U_{k}$ is the vector of all active unknowns at the node $k$. Note the dimensions of the $B$-matrix for regular nodes is $6 \times 3$, for jump-enriched nodes is $6 \times 6$, and for tip-enriched nodes is $6 \times 15$. The dimensions of the $U$ vector for regular nodes is $3 \times 1$, for jump-enriched nodes is $6 \times 1$, and for tip-enriched nodes is $15 \times 1$. For an incremental formulation, the strain increments are expressed as: $\boldsymbol{\Delta} \boldsymbol{\varepsilon}=\sum_{k=1,8} B_{k} \Delta U_{k}$ and the stresses for a small deformation problem can be written as: $\boldsymbol{\Delta} \boldsymbol{\sigma}=\boldsymbol{\sigma}_{\mathbf{0}}+$ $\boldsymbol{D} \boldsymbol{\Delta} \boldsymbol{\varepsilon}$, where $\boldsymbol{D}$ is the tangent material matrix.

The remaining part of the element formulation is quite straightforward. We assemble the $B$-matrices for the element operator: $\boldsymbol{B}=\left[B_{1}\left|B_{2}\right| \ldots \mid B_{8}\right]$ where the dimension of the $\boldsymbol{B}$ matrix is $6 \times$ total active DOFs of this element. Thus the element stiffness matrix is:

$$
\boldsymbol{K}_{\boldsymbol{e l}}=\iiint \boldsymbol{B}^{\top} \boldsymbol{D} \boldsymbol{B} \mathrm{d} x \mathrm{~d} y \mathrm{~d} z=\sum_{\alpha} \sum_{i}\left(\boldsymbol{B}^{\top} \boldsymbol{D} \boldsymbol{B}\right)_{\alpha i} w_{\alpha i} J_{\alpha}
$$

where $J_{\alpha}$ is the volume of sub-element $\alpha$; $w_{\alpha i}$ is the local weight at sampling point $i$ of the sub-element; and $\left(\boldsymbol{B}^{\top} \boldsymbol{D B}\right)_{\alpha i}$ stands for the matrix evaluated at that sampling point. The element RHS vector is given by:

$$
\boldsymbol{R}_{\boldsymbol{e l}}=\iiint \boldsymbol{B}^{\top} \boldsymbol{\sigma} \mathrm{d} x \mathrm{~d} y \mathrm{~d} z=\sum_{\alpha} \sum_{i}\left(\boldsymbol{B}^{\top} \boldsymbol{\sigma}\right)_{\alpha i} w_{\alpha i} J_{\alpha}
$$

where $\boldsymbol{\sigma}$ is the stress vector $\boldsymbol{\sigma} \triangleq\left\{\sigma_{11}, \sigma_{22}, \sigma_{33}, \sigma_{12}, \sigma_{13}, \sigma_{23}\right\}$. In Abaqus the Newton-Raphson iteration is used to resolve the nodal residuals:

$$
K_{e l} \Delta U_{e l}=F_{e l}^{e x t}-R_{e l}
$$

Here the vector of nodal unknowns $\boldsymbol{\Delta} \boldsymbol{U}_{\boldsymbol{e l}}=\left[U_{1}\left|U_{2}\right| \ldots \mid U_{8}\right]$ and $\boldsymbol{F}_{\boldsymbol{e l}}^{\boldsymbol{e x t}}$ is the vector of nodal external loads.

\section{Implicit Crack Representation and Initialization of the Level Set Representation}

In the level set representation, a 3D crack is defined by two orthogonal level set fields. One of them describes the crack surface $\{\boldsymbol{x}: \varphi(\boldsymbol{x})=0$ and $\psi(\boldsymbol{x}) \leq 0\}$, and the second is used to describe the crack front $\{\boldsymbol{x}: \varphi(\boldsymbol{x})=0$ and $\psi(\boldsymbol{x})=0\}$. This implicit description of the crack surface by level sets can be illustrated in Figure 1. 


\section{Figure 1: Illustration of the crack surface function $\varphi$ and the crack front}

The values of the two functions are generated and maintained on a narrow band of grid points around the crack surface and tip, and are used to obtain not only geometric information about the location of the crack, but also provides a local coordinate system that is used to generate the enrichment functions used in the X-FEM formulation. This is accomplished by also requiring that $\varphi$ always represents the signed distance to the extended crack surface, while $\psi$ gives the signed distance to the surface that intersects the crack surface at the crack front and is also orthogonal to the crack surface.

To initialize the values for $\varphi$ and $\psi$, we start by initializing $\varphi$ on the gridpoints of the elements that are cut by the extended initial crack surface (see circled gridpoints in Figure 2). For a given gridpoint, $\boldsymbol{x}$, the value of $\varphi(x)$ is the minimum distance between $\boldsymbol{x}$ and the crack surface elements. Assuming $\boldsymbol{y}$ is the point on a particular element where the minimum distance is obtained, and $\boldsymbol{n}$ is the unit normal to the element containing the point $\boldsymbol{y}, \varphi(\boldsymbol{x})$ is initialized by:

$$
\varphi(\boldsymbol{x})=(\boldsymbol{x}-\boldsymbol{y}) \cdot \boldsymbol{n}
$$

Once $\varphi(x)$ is computed for each gridpoint on an element cut by the crack surface, the remainder of the domain, up to the narrow band limit (see the square points in Figure 2), is initialized by using the Fast Marching Method to extend the values out to a distance $\beta$, the width of the narrow band around the crack surface as illustrated in Figure 2.

Figure 2: Illustration of the narrowband, in gray, of points around the crack surface, in cross-section, where the FMM mesh maintains signed values. The circled points are where the initialized values are computed directly and the squared points are where the values are computed using the FMM.

In this case, the values are extended

$$
\|\nabla \varphi\|=1
$$

in the remainder of the narrowband outside the directly-computed points. This extends $\varphi$, as the signed distance function, radially outward from the crack surface.

Once $\varphi$ is computed, the crack tip function, $\psi$, can now be determined so that for the crack front given as a sequence of points $\boldsymbol{y}_{m}, \psi\left(\boldsymbol{y}_{m}\right)=0$, and $\psi$ is a signed distance function such that $\nabla \psi\left(\boldsymbol{y}_{m}\right) \cdot \nabla \varphi\left(\boldsymbol{y}_{m}\right)=0$. Let $\boldsymbol{y}(s)$ be a parameterization of the crack tip, which interpolates the points $\boldsymbol{y}_{m}$, then $\psi(\boldsymbol{x})$ is computed by:

$$
\psi\left(\boldsymbol{x}_{i j k}\right)=\left(\boldsymbol{x}_{i j k}-\boldsymbol{y}(s)\right) \cdot \frac{\left(\frac{d \boldsymbol{y}}{d s} \times \boldsymbol{n}\right)}{\left\|\frac{d \boldsymbol{y}}{d s} \times \boldsymbol{n}\right\|}
$$


where $\boldsymbol{n}$ is the normal to the crack surface, the point $\boldsymbol{y}(s)$ is the point on the crack tip nearest to $\boldsymbol{x}$, and $\frac{d y}{d s} \times \boldsymbol{n}$ gives the normal to the isosurface $\psi(\boldsymbol{x})=0$. Note the values of $\varphi$ and $\psi$ are set only within a specified bandwidth, $\beta$, from the crack surface. Thus, these values are set only for grid points $\boldsymbol{x}$ such that $\psi(\boldsymbol{x}) \leq \beta$ and $|\varphi(\boldsymbol{x})| \leq \beta$.

\section{Update of the Level Set Representation}

The first step in updating the level set functions $\varphi$ and $\psi$ is to reconstruct the crack front as a one-dimensional parameterized curve from the intersection of the two isosurfaces, $\{\boldsymbol{x}: \varphi(\boldsymbol{x})=0\} \cap\{\boldsymbol{x}$ : $\psi(\boldsymbol{x})=0\}$. To do this, we start by locating a voxel that is cut by both isosurfaces, which is easily identified by a box that contains a change of sign in the values of both $\varphi$ and $\psi$ at the gridpoint vertices. Next, the point in the center of this voxel is used as an initial condition for an iterative projection onto the intersection. The iteration is given by

$$
\boldsymbol{x}^{n+1}=p\left(\boldsymbol{x}^{n}\right) \nabla p\left(\boldsymbol{x}^{n}\right)+q\left(\boldsymbol{x}^{n}\right) \nabla q\left(\boldsymbol{x}^{n}\right)
$$

where $p(\boldsymbol{x})$ is the triquintic interpolant for $\varphi(\boldsymbol{x})$, and $q(\boldsymbol{x})$ is the triquintic interpolant for $\psi(\boldsymbol{x})$. If the iteration takes $x^{n+1}$ out of the voxel, then the interpolants for the new voxel are used on subsequent iterations until it converges. Typically, only two or three iterations are required to achieve convergence, when $p\left(\boldsymbol{x}^{n+1}\right) \approx 0$ and $q\left(\boldsymbol{x}^{n+1}\right) \approx 0$. The solution of this iteration is set to be $\boldsymbol{y}_{0}$, the first point on the crack tip parameterization.

Given the point $\boldsymbol{y}_{m}$, we find $\boldsymbol{y}_{m+1}$ by first finding

$$
\overline{\boldsymbol{x}}=\boldsymbol{y}_{m}+\delta \frac{\nabla p\left(\boldsymbol{y}_{m}\right) \times \nabla q\left(\boldsymbol{y}_{m}\right)}{\left\|\nabla p\left(\boldsymbol{y}_{m}\right) \times \nabla q\left(\boldsymbol{y}_{m}\right)\right\|}
$$

where $\delta$ is the preferred approximate spacing between $\boldsymbol{y}_{m}$ and $\boldsymbol{y}_{m+1}$. The point $\boldsymbol{y}^{*}$ is then projected onto $\{\boldsymbol{x}: \varphi(\boldsymbol{x})=0\} \cap\{\boldsymbol{x}: \psi(\boldsymbol{x})=0\}$ using Eqn (20) to give $\boldsymbol{y}_{m+1}$. This continues until we close the loop by finding a point $\boldsymbol{y}_{m+1}$ such that $\left\|\boldsymbol{y}_{m+1}-\boldsymbol{y}_{0}\right\|<\delta$, or $\boldsymbol{y}_{m+1}$ is outside the computational domain. In the latter case, the crack tip is treated as an edge crack, and the crack tip must be completed by starting at $\boldsymbol{y}_{0}$ and then proceeding backward, i.e. using $-\delta$ in place of $\delta$ in Eqn (21) until the crack tip again exits the

domain. For our tests, we used $\delta=\frac{1}{2} \min (\Delta x, \Delta y, \Delta z)$, where $\Delta \mathrm{x}, \Delta \mathrm{y}, \Delta \mathrm{z}$, are the space step sizes of the mesh in each of the coordinate directions.

Once the crack tip has been parameterized, we then update $\varphi$ and $\psi$ on all grid points within a specified bandwidth distance from the crack front by direct calculation using a similar formulation to that used in Sukumar et al. (2008) except that only the narrow band region near the front is updated. In this case, given a gridpoint $\boldsymbol{x}$, we first locate the point $\boldsymbol{y}$ on the crack front nearest to $\boldsymbol{x}$ using the 
parameterized representation. Let $\boldsymbol{F}(\boldsymbol{y})$ be the crack front velocity at the point $\boldsymbol{y}$, then the new value for $\varphi$ is given by

$$
\varphi(x)=\frac{F}{\|F\|} \cdot(x-y)
$$

Once $\varphi$ is computed, $\psi$ is given by

$$
\psi(\boldsymbol{x})=\frac{(\boldsymbol{F} \times \nabla \varphi) \times \boldsymbol{F}}{\|(\boldsymbol{F} \times \nabla \varphi) \times \boldsymbol{F}\|} \cdot(\boldsymbol{x}-\boldsymbol{y})-\|\boldsymbol{F}\| \Delta t
$$

Note that the above formulas do not require the previous value to be valid, hence even if the point $\boldsymbol{x}$ may have not been in the narrow band before, it can still be easily updated. The bandwidth chosen must be large enough so that after the crack front has advanced, there are enough grid points around the new crack front location in order to populate all the data required for the triquintic interpolant around the crack front. This includes any voxel cut by the crack surface as well as a 6x6x6 array of gridpoints centered around that voxel. Thus, the bandwidth chosen will depend on the grid size, time step size, and maximum tip speed.

\section{3D Crack-Front Tracking and Growth Control Module}

In our Abaqus implementation, a key module is what used to interpolate data between the Abaqus FEM solver and the embedded level set representation. This module tracks the crack front and estimates the crack front velocity vectors based on the crack tip driving force and the fatigue law. The Abaqus user subroutine, UEXTERNALDB, is used to manage nonlocal data that cannot be accessed or updated through UEL's functional interface. These data include the nodal level set variables, element integration scheme, sub-element mesh for subsequent visualization, Gauss point coordinates, and strain/stress variables at Gauss points. They cannot fit in the existing Abaqus facilities due to the following considerations: 1) the level set variables are initialized and updated by coupling the separate level set representation, not within the user element subroutine; 2) the element slicing scheme and integration

point locations may be changed between analysis increments, whereas in the Abaqus solver the number of Gauss points and their locations are fixed throughout the whole analysis; and 3) the crack data, including tip point locations, fracture mechanics properties, crack growth velocity, and other fracture parameters, are not associated with FEM nodes or elements, therefore they cannot be stored in element-based or node-based facilities.

The user external database (UEXTERNALDB) subroutine is also used to control the analysis procedure. Before the start of analysis, level set variables are initialized to describe the initial crack geometry. At the beginning of the load increment, the crack front may advance based on the growth law; the nodal level set variables are updated using the level set representation; and subsequently the nodal enrichment type and the element slicing scheme are updated accordingly. At the end of the load increment, a post-processing module is called. The stress intensity factors at identified tip points are 
calculated; the primary and derived variables are propagated onto the sub-element mesh for visualization purposes; and the X-FEM subdomain data are saved into the Abaqus output database, or an odb file. The specific algorithms in crack front tracking and growth control are listed below:

1. Level set initialization. A structured grid for the level set representation is constructed based on the X-FEM domain min/max and the user-specified grid size $\mathrm{d} x, \mathrm{~d} y, \mathrm{~d} z$. A 3D surface mesh describing the initial crack surface, crack front, and surface boundary is used to initialize the level set values at grid nodes. Another surface mesh describing the free surfaces, or the skin of the X-FEM element region, is used to identify the active level set region by intersecting the level set grid domain. Only the level set variables in the active region of the level set domain are initialized and subsequently updated. The level set initialization and updates are discussed in Sections 3, 4.

2. Crack growth control. The stress intensity factors $\left(K_{\mathrm{I}}, K_{\mathrm{II}}\right.$, and $\left.K_{\mathrm{III}}\right)$ are estimated with respect to a local coordinate system $\left\{e_{1}, e_{2}, e_{3}\right\}$ at a tip point, such that $e_{1}$ is the normal to the tangent surface of the crack, and $e_{2}$ is on the tangent surface and normal to the crack front curve. The crack growth direction vector, $\Delta a$, is determined as follows. The growth is assumed to be on the $\left\{e_{1}, e_{2}\right\}$ plane and the angle $\theta$ between $\Delta a$ and $e_{2}$ is determined such that the stress intensity factor $K_{\mathrm{I}}$ is maximum. This is consistent with the maximum hoop direction which can be expressed as:

$$
\theta=2 \operatorname{atan}\left[\frac{1}{4}\left(\frac{K_{\mathrm{I}}}{K_{\mathrm{II}}} \pm \sqrt{\left.\left(\frac{K_{\mathrm{I}}}{K_{\mathrm{II}}}\right)^{2}+8\right)}\right] .\right.
$$

The crack growth size, $\Delta a$, is computed from numerical integration of the Paris law

$$
\Delta=\int_{\Delta N} C\left(\Delta K-\Delta K_{\mathrm{th}}\right)^{m} \mathrm{~d} N
$$

where $C$ and $m$ are fracture parameters, $\Delta K_{\text {th }}$ is the threshold depending on materials, and $\Delta K$ is the range of equivalent $K$ during a load cycle. In the presence of mean stress and nonzero load ratio, the Walker's equation is used instead, where $\Delta K$ is replaced by

$$
\Delta K_{\text {walker }}=\underset{(1-R)^{1-\gamma}}{2 \Delta},
$$

where $R$ is the load ratio, and $\gamma$ is a material constant which can be calibrated from da-dN curves at different $R$ ratios. For a 3D crack, multiple sampling tip points are selected along the front and denoted by $T_{i}^{*}$. It is necessary to adjust $\Delta a_{i}$, which corresponds to tip $T_{i}^{*}$, such that $\Delta N$ is consistent for all tip points. For constant amplitude loading an explicit formula can be used to adjust $\Delta a_{i}$. In this case we first identify the sampling point $T_{\max }^{*}$ that possesses the maximum $\Delta K . \Delta N$ is then back-calculated using the Paris law based on the user-specified $\Delta a_{\max }$. Different from a conventional approach where $\Delta N$ is used for the update, we choose $\Delta a_{\max }$ as the controlling parameter of the analysis procedure because of the freedom of crack growth without conformation to the existing mesh. In addition, the accelerated crack growth behavior near the final failure stage may result in a large crack growth step size under a predefined $\Delta N$. With a predefined $\Delta a_{\max }, \Delta a_{i}$ at all tip points along the crack front can be estimated by:

$$
\Delta a_{i}=\left(\frac{\Delta K_{i}-\Delta K_{\mathrm{th}}}{\Delta K_{\max }-\Delta K_{\mathrm{th}}}\right)^{m} \Delta a_{\max } .
$$


The crack tracking and update can be illustrated in Figure 3:

Figure 3: Overview of crack tracking and growth control.

3. Nodal updates. The crack growth vectors, $\Delta a$, and the coordinates of the tip points where $\Delta a$ are computed, are required by the embedded level set representation to update the level set variables in the active region. The tip points are selected at the mid-points of the crack line segments connecting the two intersection points of the tip element boundary and the crack front. After update of the level set values, $\varphi$ and $\psi$, they are interpolated to the FEM nodes. The shortest distance from an FEM node to the front can then be recovered as $\sqrt{\varphi^{2}+\psi^{2}}$. The tip enrichment is assigned to the nodes that are closer to the front than a user-specified value. All the nodes of an element partially cut by the crack surface are tip-enriched to ensure the partition of unity and reproduction of the asymptotic field for those elements. For the nodes that are not within the tip enrichment zone but are connected to an element completely cut by the crack surface, jump function enrichment is assigned to those nodes.

4. Allocation of CTOD displacement sampling points. It is convenient to estimate displacement jumps at a point on the crack surface using:

$$
\begin{aligned}
\llbracket U(x, y, z) \rrbracket= & \llbracket u(\xi, \eta, \zeta) \rrbracket= \\
& \sum_{i} N_{i}(\zeta, \eta, \xi) \llbracket H\left(x^{+}, y^{+}, z^{+}\right)-H\left(x^{-}, y^{-}, z^{-}\right) \rrbracket b_{i}+ \\
& \sum_{i} N_{i}(\zeta, \eta, \xi) \llbracket \Psi_{1}\left(x^{+}, y^{+}, z^{+}\right)-\Psi_{1}\left(x^{-}, y^{-}, z^{-}\right) \rrbracket c_{1 i}
\end{aligned}
$$

where $(x, y, z)$ and $(\xi, \eta, \zeta)$ are global and local coordinates of the sampling point; $\left(x^{+}, y^{+}, z^{+}\right)$ and $\left(x^{-}, y^{-}, z^{-}\right)$are coinciding points associated with to the positive and negative surfaces of a crack, respectively. By substituting the following equations into Eqn (28):

$$
\begin{aligned}
& H\left(x^{+}, y^{+}, z^{+}\right)=+1, H\left(x^{-}, y^{-}, z^{-}\right)=-1, \\
& \Psi_{1}\left(x^{+}, y^{+}, z^{+}\right)=\sqrt{\mathrm{r}} \sin \left(\frac{\pi}{2}\right), \Psi_{1}\left(x^{-}, y^{-}, z^{-}\right)=\sqrt{\mathrm{r}} \sin \left(-\frac{\pi}{2}\right),
\end{aligned}
$$

we obtain:

$$
\llbracket u(\xi, \eta, \zeta) \rrbracket=\mathbf{2} \sum_{i} N_{i}(\zeta, \eta, \xi)\left(b_{i}+\sqrt{r} c_{1 i}\right)
$$

Note that for points on the crack surface behind the front the orientation angle $\theta= \pm \pi$. From the above equation it is seen to be convenient to allocate the CTOD sampling points through an element loop because transferring from a local coordinate system to the global coordinate system is much easier. The specific algorithm to identify the CTOD elements is the following:

1) Find the candidate elements with nodal level set $\psi_{\min }<-r<\psi_{\max }$, where $r$ is the offset distance from the tip where displacement jumps are censored for CTOD;

2) Select elements that actually contain a segment of the $\psi=-r$ surface and a segment of $\varphi=0$ 
3) On the two element facets that contain the intersection points of the curve: $\psi=-r \cap \varphi=0$, find the local coordinates of points on $\psi=-r$ using the gradient-based search on the bilinear surface:

$$
\psi\left(\xi^{\prime}, \eta^{\prime}\right)=\sum_{i=1,4} N_{i}\left(\xi^{\prime}, \eta^{\prime}\right) \Psi_{i}
$$

where $\Psi_{i}, i=1 \sim 4$ are nodal $\Psi$ values of the concerned facet and $\left(\xi^{\prime}, \eta^{\prime}\right)$ are the two varying coordinates on the facet (with the third coordinate being constant: -1 or +1 ). These two intersection points are designated by tri-variant reference coordinates as $P_{1}\left(\xi_{1}, \eta_{1}, \zeta_{1}\right)$ and $P_{2}\left(\xi_{2}, \eta_{2}, \zeta_{2}\right)$. Note two of the triple $(\xi, \eta, \zeta)$ are $\xi^{\prime}, \eta^{\prime}$ and the third one is \pm 1 . Find the global coordinates of $P_{1 / 2}(x, y, z)$ using the following shape function interpolation:

$$
\begin{aligned}
& x(\xi, \eta, \zeta)=\sum_{i} N_{i}(\xi, \eta, \zeta) X_{i}, y(\xi, \eta, \zeta)=\sum_{i} N_{i}(\xi, \eta, \zeta) Y_{i}, \\
& z(\xi, \eta, \zeta)=\sum_{i} N_{i}(\xi, \eta, \zeta) Y_{i}
\end{aligned}
$$

Now, select the CTOD censoring point as the midpoint of the line segment: $P_{1}-P_{2}$, or $P^{*}\left(\frac{x_{1}+x_{2}}{2}, \frac{y_{1}+y_{2}}{2}, \frac{z_{1}+z_{2}}{2}\right)$.

4) Follow the similar approach in Step (3), except that the cylinder is now $\psi=0$ and the intersection points $T_{1}(x, y, z)$ or $T_{2}(x, y, z)$ are the tip points on curve: $\psi=0 \cap \varphi=0$;

5) Project $P^{*}$ onto the crack front segment: $T_{1}-T_{2}$ and designate the projected point as $T^{*}$;

6) Define a coordinate system $\left\{e_{1}, e_{2}, e_{3}\right\}$ that is consistent to the local fracture mode decomposition and attach it to the tip point $T^{*}$, such that $e_{1}$ is a unitary vector parallel to the crack surface normal direction, $e_{2}$ is a unitary vector parallel to the tip growth direction, or $P^{*} \rightarrow T^{*}$, and $e_{3}$ is also a unitary vector parallel to the tangent direction of the front curve: $T_{1}-T_{2}$. The global to local transformation matrix $\boldsymbol{R}$ can be defined as:

$$
\boldsymbol{R}=\left[\begin{array}{lll}
\mathrm{X}_{\mathrm{e}_{1}} & \mathrm{X}_{\mathrm{e}_{2}} & \mathrm{X}_{\mathrm{e}_{3}} \\
\mathrm{Y}_{\mathrm{e}_{1}} & \mathrm{Y}_{\mathrm{e}_{2}} & \mathrm{Y}_{\mathrm{e}_{3}} \\
\mathrm{Z}_{\mathrm{e}_{1}} & \mathrm{Z}_{\mathrm{e}_{2}} & \mathrm{Z}_{\mathrm{e}_{3}}
\end{array}\right]
$$

7) Finally, it is important to adjust the actual offset distance from its nominal value of $r$ to the true distance between $P^{*}$ and $T^{*}$.

5. Update of element integration scheme. For elements that are cut partially or completely by a crack surface, a new slicing scheme is needed to reflect crack advance. The details of element slicing are discussed in Section 7.

6. Post-processing for fracture mechanics analysis. At the element level, compute the displacement jumps $\llbracket u(\xi, \eta, \zeta) \rrbracket$, where $(\xi, \eta, \zeta)$ are reference coordinates of the sampling point $P^{*}$. At the system level, once the iteration is converged, transform $\llbracket u \rrbracket$ into the local coordinate system using: $R \llbracket u \rrbracket=\left(U_{\mathrm{I}}, U_{\mathrm{II}}, U_{\mathrm{III}}\right)$. Compute the stress intensity factors:

$$
K_{\mathrm{I}}=\frac{\mu \sqrt{2 \pi / r}}{\kappa+1} U_{\mathrm{I}}, \quad K_{\mathrm{II}}=\frac{\mu \sqrt{2 \pi / r}}{\kappa+1} U_{\mathrm{II}} \text {, and } K_{\mathrm{III}}=\frac{\mu \sqrt{2 \pi / r}}{2} U_{\mathrm{III}} .
$$

For fatigue life prediction under multi-axial loading, the following equivalent stress intensity factor, 
$K_{\text {eqv }}$, is also computed:

$$
K_{\mathrm{eqv}}=\sqrt{K_{\mathrm{I}}^{2}+K_{\mathrm{II}}^{2}+\left(1-v^{2}\right) K_{\mathrm{III}}^{2}}
$$

In Eqn (35) $\mu$ and where $\kappa$ are defined by $\mu=\frac{E}{2(1+v)}, \kappa=3-4 v$ for the plain-strain assumption or $\kappa=(3-v) /(1+v)$ for the plain-stress assumption, respectively; $v$ is Possion's ratio, $E$ is the Young's modulus, and $r$ is the true distance from the sampling point $P^{*}$ to its corresponding tip point $T^{*}$. To avoid direct calculation of $K_{\text {eqv }}$ in cyclic loading, the linear scaling of $K_{\text {eqv }}$ is used such that $\Delta K=K_{\text {eqv }} \frac{P_{\max }-P_{\min }}{P_{\text {ref }}}$, where $P_{\max }$ and $P_{\min }$ are the external loads at the turning points. To accurately capture the non-self-similar and non-planar growth pattern of a 3D evolving crack, the crack growth driving force $\left\{\Delta K_{i}\right\}$ is computed at a set of predefined sampling points $\left\{T_{i}^{*}\right\}$. The computed $\left\{\Delta K_{i}\right\}$ are then used to determine the growth velocity vectors defined in Eqn (27) of Step 2 to track its growth.

\section{Penalty Approach for Contact and Friction}

Residual stresses and near tip plasticity may cause crack close during the growth process. Sliding and frictional contact are important aspects in computing the crack tip driving force for crack onset and growth prediction. Such contact interactions can be described by the principle of virtual work in the following equilibrium equation:

$$
\int_{\Omega} \sigma: \delta \varepsilon \mathrm{d} v+\oint_{S} \tau \cdot \delta \llbracket u \rrbracket \mathrm{d} s=\int_{\Omega} f \cdot \delta u \mathrm{~d} v+\oint_{\partial \Omega} \tau \cdot \delta u \mathrm{~d} s \text { with } \llbracket u \rrbracket \cdot n \geq 0
$$

where $\llbracket u \rrbracket$ is the displacement jump defined in Eqn (30), $n$ is the crack surface normal direction, and $\oint_{S} \tau \cdot \delta \llbracket u \rrbracket \mathrm{d} s$ is the virtual work associated with the surface interaction. This surface interaction term may be denoted by $\delta \pi_{I N T}$ and is usually presented in the local coordinate system defined by the front tangential and surface normal at the front, ie.,

$$
\delta \pi_{\mathrm{INT}}=\oint_{\bar{S}} \tilde{\tau} \cdot \delta \Delta \mathrm{d} \bar{s}
$$

In Eqn (37) $\tilde{\tau}$ is the surface traction in the local coordinate system; $\delta \Delta$ contains the opening displacements along the normal and two sliding directions and it relates to $\llbracket u \rrbracket$ by $\delta \Delta=R \delta \llbracket u \rrbracket$, where $R$ is defined in Eqn (33). Using Eqn (30), if we introduce $B_{i}^{S}=R N_{i}[2,2 \sqrt{r}]_{i}$, then the opening

displacements can be expressed by $\delta \Delta=\sum_{i} B_{i}^{S} \delta\left[b, c_{1}\right]_{i}{ }^{\top}$. Applying the Gauss quadrature in Eqn (37), we get: 


$$
\delta \mathrm{d} \pi_{\mathrm{INT}}=\oint_{\bar{S}} \mathrm{~d} \tau \cdot \delta \Delta \mathrm{d} \bar{s}=\sum_{l} s_{l} \mathrm{~d} \tau_{l} \delta \Delta_{l}
$$

where $s_{l}$ is surface area associated with the contact integration point, $l, \tau_{l}$ refers to the reaction force, and $\Delta_{l}$ represents to the displacement jumps at this integration point. The traction force $\tau_{l}$ contains both the contact reaction force and the frictional force, and is related to $\Delta_{l}$ as:

$$
\tau_{l}=D^{s} \Delta_{l}=\left[\begin{array}{ccc}
-K_{1} & 0 & 0 \\
\mu K_{1} & K_{2} & 0 \\
\mu K_{1} & 0 & K_{3}
\end{array}\right]\left[\begin{array}{c}
\Delta_{l, 1} \\
\Delta_{l, 2} \\
\Delta_{l, 3}
\end{array}\right]
$$

where the penalty stiffness $K_{1}$ ramps down to zero when $\Delta_{l, 1} \geq-h_{c r}$, and $h_{c r}$ is the critical penetration displacement across the surface; $K_{2}$ or $K_{3}$ also ramps down to zero when $\Delta_{l, 2}$ or $\Delta_{l, 3} \geq$ $\Delta_{c r}$ respectively, and $\Delta_{c r}$ is the critical elastic slip after which the contact surfaces start to slide; and $\mu$ is the Coulomb friction parameter. Now the surface interaction term $\delta \mathrm{d} \pi_{I N T}$ becomes:

$$
\delta \mathrm{d} \pi_{\mathrm{INT}}=\sum_{l} s_{l} \sum_{i} \delta\left[b, c_{1}\right]_{i} B_{i}^{s^{\top}} D^{s} B_{i}^{s} \mathrm{~d}\left[b, c_{1}\right]_{i}^{\top}=\sum_{l} s_{l} \boldsymbol{\delta} \boldsymbol{U}^{\top}\left(\boldsymbol{B}^{\boldsymbol{s}^{\top}} \boldsymbol{D}^{\boldsymbol{s}} \boldsymbol{B}^{\boldsymbol{s}}\right)_{l} \mathbf{d} \boldsymbol{U}
$$

This term contributes to the element stiffness as the penalty stiffness matrix and the penalty force vector:

$$
\boldsymbol{K}^{\boldsymbol{s}}=\sum_{l} s_{l}\left(\boldsymbol{B}^{\boldsymbol{s}^{\top}} \boldsymbol{D}^{\boldsymbol{s}} \boldsymbol{B}^{\boldsymbol{s}}\right)_{l} \text { and } \boldsymbol{F}^{\boldsymbol{s}}=-\boldsymbol{K}^{\boldsymbol{s}} \boldsymbol{U}
$$

The penalty contact algorithm is summarized below. For each equilibrium iteration:

1) Identify the surface segment that is in contact; calculate the global - local transformation matrix $\boldsymbol{R}$.

2) Allocate integration points along the contact surface; obtain the coordinates of the integration points in the local coordinate system; calculate the surface area $S_{l}$.

3) Calculate the surface $B$-matrix $\boldsymbol{B}^{\boldsymbol{S}}$ at the integration points based on Eqn (40).

4) Calculate the total displacement jumps $\Delta=\sum_{i} B_{i}^{s}\left[b, c_{1}\right]_{i}^{\top}=\boldsymbol{B}^{\boldsymbol{s}} \boldsymbol{U}$;

5) If $\Delta_{l, 1} \geq-h_{c r}$, then calculate the penalty stiffness matrix $\boldsymbol{K}^{\boldsymbol{s}}$ and nodal penalty force $\boldsymbol{F}^{\boldsymbol{s}}$ based on Eqn (41), and then assemble the corresponding terms to the element stiffness matrix $\boldsymbol{K}_{\boldsymbol{e l}}$ in Eqn (14) and the vector of right-hand-side $\boldsymbol{R}_{\boldsymbol{e l}}$ in Eqn (15).

\section{Slicing Schemes for Numerical Integration of Completely or Partially Cut Elements}

For an element being cut by a crack surface, its volume should be divided into sub-regions inside which the displacements are continuous functions, so that the nodes and weights of Gauss quadrature rules can be computed for each sub-region. Distinct from local remeshing schemes, the volumetric integration is performed at the element level, not its sub-elements. Slicing scheme was introduced in Sukumar et al. (2000) for an element completely cut by a bilinear crack surface.

In this section the slicing scheme is naturally extended to the partial cut cases shown in Figure 4. The algorithm for complete cut cases as in Figure 4(a) can be summarized as follows: 1) the intersected 
facets of an element are firstly identified by the intersection points $c_{1}$ and $c_{2} ; 2$ ) the facets are then sliced into triangles by connecting corner nodes $k_{1}, k_{2}, k_{3}$ or $k_{4}$, intersection points $c_{1}$ or $c_{2}$, and the area centers of either sub-region; and 3) the tetrahedral sub-elements of the underlined brick element are obtained by connecting the triangles and the volumetric centers of either sub-volume. For partially cut cases, the crack surface is extended tangentially to the element facets. For the facets that are cut completely by the extended surface, the same triangulation scheme in Figure 4(a) is adopted, while for the other facets cut by both original and extended surfaces, a different triangulation scheme in Figure 4(b) is used. In this scheme the area centers are skipped and all triangles connect to the crack tip.

\section{Figure 4: Slicing scheme on the quad facet: (a) scenarios of facets being completely cut by a crack surface represented by the line $c_{1}-c_{2}$; and (b) scenarios of facets partially cut by a crack surface.}

The element slicing is performed once until the next change due to mesh interactions. Therefore only the elements inside the tip enrichment zone are checked against re-slicing when the intersecting crack advances, including the partially cut elements and the unpartitioned elements that connect to any of the tip enriched nodes. Double nodes are created for the intersections points, $c_{1}$ and $c_{2}$ in scheme (a) and $c_{2}$ in scheme (b) and the area center in scheme (b) that are on the crack surface and behind the tips. For these double nodes, the $\varphi$ level set values are either $0^{+}$or $0^{-}$in order to reserve the sign that designates the association of the nodes to the positive crack surface or negative crack surface, respectively.

\section{SPR State Mapping for Dynamically Allocated Material Point Integration}

The element slicing scheme given in Sukumar et al. (2000) is adopted in our implementation because it represents exact volume integration with $\mathrm{O}\left(\mathrm{h}^{\mathrm{p}+1}\right)$ polynomials with much fewer Gauss points. The only disadvantage of such slicing is that the integration points are dynamically allocated from one load increment to another. This is not an issue for elastic simulation if one uses a total strain-stress formulation. In the case of crack propagation in elastic-plastic fracture mechanics, material point behavior depends on the load history and an incremental form of material constitutive models has to be used. The recovery of material state variables, or projection of stress and plastic flow onto new integration points, cannot be avoided. In order to resolve this issue, (Elguedj et al., 2006) has proposed a hybrid approach consisting of a second set of static integration points to keep track of the plastic flow. That approach requires $256+256$ integration points for a 2D quad element stiffness generation; therefore it would be too computationally intensive for a practical 3D problem.

There are several state mapping algorithms, including: 1) element oriented interpolation using shape functions, typically used in visualization modules; 2) classical L2 projection which was widely used in the 70's; and 3) the patch-oriented transfer super-convergent patch recovery, or SPR, in Zienkiewicz-Zhu (1992) which obtains a uniform $\mathrm{O}\left(\mathrm{h}^{\mathrm{p}+1}\right)$ accuracy of stress. Unlike the L2 method 
where the accuracy degenerates near the element boundary, in both cases the SPR can recover the stress to an order higher than the shape function uniformly in the element domain.

To enhance the numerical efficiency, a super-convergent patch recovery (SPR) mapping is developed for dynamic allocation of Gauss points. During the construction step, the state components at the interpolating nodes are estimated using a least square fitting from the neighboring Gauss points. Under the recovery step, the states at the new Gauss points are obtained by shape function interpolation in the underlined tetrahedron sub-element.

The algorithm for the construction step is described as follows. Assume that a nodal state $s_{N}$ can be interpolated by a polynomial function, $s_{p}\left(x_{k}, y_{k}, z_{k}\right)$ where $p$ denotes the polynomial order. We have:

$$
s_{p}\left(x_{k}, y_{k}, z_{k}\right)=\boldsymbol{P}\left(x_{k}, y_{k}, z_{k}\right) \boldsymbol{a}
$$

where:

$$
\begin{aligned}
& \boldsymbol{P}\left(x_{k}, y_{k}, z_{k}\right)=\left[1, x_{k}, y_{k}, z_{k}, x_{k}{ }^{2}, y_{k}{ }^{2}, z_{k}{ }^{2}, x_{k} y_{k}, x_{k} z_{k}, y_{k} z_{k} \ldots\right], \text { and } \\
& \boldsymbol{a}=\left[a_{0}, a_{1}, a_{2}, a_{3}, a_{11}, a_{22}, a_{33}, a_{12}, a_{12}, a_{23} \ldots\right]^{\top}
\end{aligned}
$$

The coefficients $\boldsymbol{a}$ are found via the least square fitting from high accuracy sampling points $\mathrm{s}_{h}$ (Gauss points), or by solving the linear equation

$$
\sum_{k} \boldsymbol{P}\left(x_{k}, y_{k}, z_{k}\right)^{\top} \boldsymbol{P}\left(x_{k}, y_{k}, z_{k}\right) \boldsymbol{a}=\sum_{k} \boldsymbol{P}\left(x_{k}, y_{k}, z_{k}\right)^{\top} \mathrm{s}_{h}\left(x_{k}, y_{k}, z_{k}\right)
$$

where the subscript $k$ indicates the Gauss points being used for this fitting. The nodal state $s_{i}$ is then interpolated by

$$
\mathrm{s}_{i}\left(x_{N}, y_{N}, z_{N}\right)=\boldsymbol{P}\left(x_{N}, y_{N}, z_{N}\right) \boldsymbol{a}
$$

Note that the state $\mathrm{s}_{i}$ is recovered for all sub-nodes, $i$, in X-FEM elements containing active crack tips.

For the recovery step, the state at a new Gauss point is determined by:

$$
s^{*}=\sum_{i} N_{i}\left(\xi^{*}, \eta^{*}, \zeta^{*}\right) s_{i}
$$

where $N_{i}$ is the shape function associated with sub-node $i$.

The matrix inversion involved in Eqn (44) is only performed once per element regardless of the number of nodes of an element; propagating the state to nodes and interpolation to new Gauss points are essentially linear transformation and takes $O$ (number of nodes per element) operations. Most FEM nodes belong to several patches and different values of $s_{I}$ will be available. Each of these values is super-convergent and a simple averaging process gives generally the best approximation. In performing the least square fitting operation locally based coordinate origins should be used to avoid ill-conditioning. This is particularly important for $p>2$ (in near-tip elements $p$ as high as 10 is used).

\section{3D X-FEM Validation Examples}

In this section, the published data for standard and modified compact test specimens (miss-hole 
configuration vs. sink-hole configuration) are utilized to evaluate the proposed method. These examples study the accuracy of predictions of stress intensity factors, curvilinear crack paths, and fatigue life. Mesh sensitivity study and parametric study on several X-FEM control parameters are also performed.

\subsection{Validation of stress intensity factors using a standard compact specimen}

The first example shown in Figure (5) features a standard compact test specimen subjected to uniaxial tension. It is used to validate stress intensity factor prediction by comparing to the analytical solution of Lei (2006). Specimen geometry and material properties are adopted from Lei (2006). The width of specimen is of $38.2 \mathrm{~mm}$, height $45.84 \mathrm{~mm}$, thickness $15.35 \mathrm{~mm}$, and the distance of tip from the center of loading holes $21.01 \mathrm{~mm}$. The material is a $316 \mathrm{H}$ steel. An elasto-plastic material model is employed, with $E=160 \mathrm{GPa}, E_{1}=1,466 \mathrm{MPa}, \sigma_{y}=234 \mathrm{MPa}$, and Poisson's ratio $v=0.28$. A crack of length $a=21.01 \mathrm{~mm}$ is inserted. A vertical tension load of $11 \mathrm{kN}$ is applied to create a pure Mode I case. The geometry of the specimen and the mesh design is shown in Figure 5. We apply a refined mesh near the tip region. There are six elements along the specimen width direction.

Figure 5: $K$ validation using a standard compact test specimen. (a) Specimen dimensions and X-FEM mesh design; (b) von Mises stresses in deformed shape.

The analytical solution given by Lei (2006) for plane strain is:

$$
K=\frac{P}{B W^{\frac{1}{2}}} \frac{2+\frac{a}{W}}{\left(1-\frac{a}{W}\right)^{\frac{3}{2}}} f\left(\frac{a}{W}\right),
$$

where $f\left(\frac{a}{W}\right)=0.886+4.64 \frac{a}{W}-13.32\left(\frac{a}{W}\right)^{2}+14.72\left(\frac{a}{W}\right)^{3}-5.6\left(\frac{a}{W}\right)^{4}$

It gives $K=1317.63 \mathrm{MPa} \mathrm{mm}^{1 / 2}$ for the geometry of our problem.

Several combinations of X-FEM parameters were used; and the K values along the crack front direction are plotted in Figure 6. Note that since we have six elements along the width, six data points for each combination of parameters are obtained. They are plotted in a dimensionless form relative to the plane strain solution $K=1317.63 \mathrm{MPa} \mathrm{mm}{ }^{1 / 2}$. The characteristic near-tip element size is $0.35 \mathrm{~mm}$. We choose the enrichment radius as 1) $0.3 \mathrm{~mm}$, that is, only the nodes shared by the partially cut elements are enriched by branch functions, 2) $0.55 \mathrm{~mm}$ with two layers of elements surrounding the crack front being tip-enriched, and 3) $0.75 \mathrm{~mm}$ with three layers of tip enriched elements. Because of the embedded singular field in the branch functions, the offset distance, $r$, in CTOD should be less dependent on the $\mathrm{K}$ estimates as indicated in Eqn (35). We select $r$ from $0.0001 \mathrm{~mm}$ to $0.90 \mathrm{~mm}$. These results confirm that for all tests with enrichment radius more than twice of the element size and the CTOD offset distance less than enrichment radius, the $\mathrm{K}$ predictions are within $5 \%$ error by comparing to the reference solution. Note that in using the CTOD, the plane strain solution is used to back-calculate the stress intensity factor. 
Figure 6: (a) Dimensionless $K$ values in reference to Eqn (47) using X-FEM with a combination of parameter settings; and (b) relative errors in $K$ prediction as a function of the tip enrichment radius and the CTOD offset distance.

\subsection{Mesh sensitivity study of a miss-hole compact test specimen problem}

This second example considers a modified compact tension (CT) specimen in Miranda et al. (2003) with an additional hole in the standard CT specimen. The CT specimen geometry is detailed in Figure 7 (unit: millimeter). The presence of the additional hole will perturb the crack path, resulting in a curvilinear crack growth. In the first case we consider the miss-hole configuration with the hole position of $\mathrm{A}=8.3 \mathrm{~mm}$ and $\mathrm{B}=8.1 \mathrm{~mm}$. The material is a cold rolled SAE 1020 steel. The material properties are: Young's modulus $E=205$, yield strength $\sigma_{y}=285 \mathrm{MPa}$ and the ultimate strength $\sigma_{u}=491 \mathrm{MPa}$. The geometry of the specimen is shown in Figure 7. For the fatigue crack growth prediction, the Paris law is used with the parameters $C=4.5 \mathrm{E}-10, m=2.1$ as defined in Eqn (25), and the fatigue growth threshold value $\Delta K_{\mathrm{th}}=11.6 \mathrm{MPa} \sqrt{\mathrm{m}}$.

Figure 7: Geometry of the modified CT specimen. $A=8.3 \mathrm{~mm}$ and $B=8.1 \mathrm{~mm}$ for the miss-hole configuration; and $A=8.4 \mathrm{~mm}$ and $B=6.9 \mathrm{~mm}$ for the sink-hole configuration.

We used two meshing schemes: a free mesh, or an unstructured mesh, and a structured mesh. In this case we use one element through the depth. The mesh design, the overall crack growth path, and the von Mises stress distribution at the final configurations are shown in Figure 8. Note that for both models the smallest characteristic element size is about $0.3 \mathrm{~mm}$. An almost identical crack path and stress profile are obtained using both the free and structured mesh.

Figure 8: Mesh sensitivity study of crack growth patterns for the miss-hole CT specimen under constant cyclic loading

In Figure 9 we plot $K_{\mathrm{I}}$ (Figure 9-a) and $K_{\mathrm{II}}$ (Figure 9-b) as functions of the crack extension size in $\mathrm{mm}$. Results from several combinations of parameters are plotted:

1) Free mesh with crack growth size $d a=0.3 \mathrm{~mm}$, enrichment zone size $r=0.75 \mathrm{~mm}$, COTD offset distance $l=0.75 \mathrm{~mm}$;

2) Free mesh with crack growth size $d a=1.0 \mathrm{~mm}$, enrichment zone size $r=0.90 \mathrm{~mm}$, COTD offset distance $l=0.75 \mathrm{~mm}$;

3) Structured mesh with crack growth size $d a=1.0 \mathrm{~mm}$, enrichment zone size $r=0.90 \mathrm{~mm}$, COTD offset distance $l=0.75 \mathrm{~mm}$;

4) Free mesh with crack growth size $d a=0.5 \mathrm{~mm}$, enrichment zone size $r=0.75 \mathrm{~mm}$, COTD offset 
distance $l=0.01 \mathrm{~mm}$; and

5) Structured mesh with crack growth size $d a=0.5 \mathrm{~mm}$, enrichment zone size $r=0.75 \mathrm{~mm}$, COTD offset distance $l=0.01 \mathrm{~mm}$.

Figure 9: Stress intensity factors $K_{I}(a)$ and $K_{I I}(b)$ as functions of crack extension size, using combinations of $\mathrm{X}$-FEM analysis controlling parameters.

Both $K_{\mathrm{I}}$ and $K_{\mathrm{II}}$ solutions agree reasonably well with each other, regardless the use of different X-FEM analysis controlling parameters. Note that ideally $K_{\mathrm{II}}$ should be zero because the mode I direction is assumed as the crack growth direction shown in Eqn (24). However, since the direction is explicitly determined, or the direction for the next time increment is determined by the $K_{\mathrm{I}}$ and $K_{\mathrm{II}}$ values from the previous increment, the $K_{\text {II }}$ can be a rather small positive or negative value as the crack grows, depending on the selected crack growth size. A larger numerical noise in $K_{\text {II }}$ indicates that a smaller growth size may be needed.

Figure 10 plots the crack profiles (Figure 10-a) and the fatigue life prediction (Figure 10-b) under a constant amplitude cyclic load. All the five cases shown in Figure 9, along with one additional free-mesh case: 6 ) Free mesh with crack growth size da $=1.0 \mathrm{~mm}$, enrichment zone size of $0.75 \mathrm{~mm}$, COTD offset distance of $0.01 \mathrm{~mm}$, and an 11-Gauss-point integration scheme used for all sub-elements after the element slicing. Note among all other cases only the partially-cut elements use 11-Gauss-point integration for their sub-elements. For the elements sharing a tip-enriched node, the 4-Gauss-point integration is used, and for the elements sharing a jump-enriched node, the 1-Gauss-point integration is used. For uncut brick elements not connecting to any tip-enriched nodes, the full integration using 8-Gauss-point integration is used. As shown in Figure 10, the prediction on the crack paths and life curve $a(N)$ are similar with a small deviation.

Figure 10: Crack profile (a) and fatigue life prediction (b) as functions of crack extension size, using combinations of $\mathrm{X}$-FEM analysis controlling parameters.

\subsection{Validation of fatigue life prediction using the modified CT specimens: miss-hole configuration and sink-hole configuration}

To compare with the experiment data presented in Miranda et al. (2003), the same applied load curve is employed such that the resulting SIF range is constant: $\Delta \mathrm{E}=20 \mathrm{MPa} \sqrt{\mathrm{m}}$. The actual applied load for both hole configurations is interpolated from data shown in Miranda et al. (2003) and then used in the analyses, which ranges from about $750 \mathrm{kN}$ to $225 \mathrm{kN}$ (Figure 11).

Figure 11: Loading history for the modified-hole CT specimen analyses. 
The predicted $K_{\mathrm{I}}$ by X-FEM is substituted back into the equation: $f\left(\frac{a}{w}\right)=\frac{K_{I} w t}{P \sqrt{\pi a}}$ to calculate $f\left(\frac{a}{w}\right)$ and compared with the prediction in Miranda et al. (2003). The calculated $f(a / w)$ from X-FEM are plotted with the reference solution in Figure 12. The X-FEM prediction matches the reference curves very well. This excellent agreement further verifies the accuracy of X-FEM in predicting the $K_{\mathrm{I}}$ and $K_{\mathrm{II}}$ for a growing curvilinear crack.

Figure 12: Comparison of calculated $f(a / w)$ for the modified-hole specimens.

The $a(N)$ curves prediction for both sink-hole and miss-hole cases are compared with the experimental data in Figure 13. Note that the $a(N)$ curves should be close to linear since the tests are controlled under a constant $\Delta \mathrm{E}=20 \mathrm{MPa} \sqrt{\mathrm{m}}$. For the sink-hole case, the X-FEM prediction curve agrees well with the experiment measurements. For the miss-hole case, the model slightly underestimates the fatigue life for a given crack length $a$ due to the intrinsic variability in fatigue testing results. Despite this, the overall trend is still in good agreement with the test data.

Figure 13: Comparison of fatigue life prediction for the modified-hole specimens

Several snapshots on crack growth path at different number of cycles are shown in Figure 14 for both miss-hole and sink-hole configuration.

Figure 14: Snapshots of CT specimen von Mises stress as crack grows.

Finally, the X-FEM crack path predictions are plotted on the digital images of the actual test specimens as shown in Figure 15. The measured crack paths for both the sink and miss case are in excellent agreement with the corresponding XFEM prediction.

Figure 15: Comparison of crack path prediction: miss-hole (CT1) on the left and sink-hole (CT2) on the right.

\section{Demonstration Example: Crack Growth and Fatigue Life Predictions in a Complex Helicopter Component under Spectrum Loadin}

To further demonstrate the current 3D X-FEM toolkit for fatigue life prediction of a structural component subjected to spectrum loading, a round-robin problem provided by Airbus/UK is considered here. The problem has also been studied by Newman et al. (2006). Two solution steps are used to 
simulate the fatigue crack growth and remaining life under spectrum loading: 1) use of the 3D X-FEM toolkit to determine the functional relation between the crack length and its associated stress intensity factors; and 2) perform the post fatigue life prediction using the AFGROW (Harter, 2008) originally developed by the Air Force Research Laboratory.

The selected geometry is a flanged plate with a central lightening hole as shown in Figure 16. This is representative of many features found in a helicopter lift frame. Crack growth would be initiated from a corner defect, $a=b=2.0 \mathrm{~mm}$ on the inner edge of the hole. The selected material is aluminum alloy of AA 7010 T73651. Comprehensive constant amplitude fatigue crack growth rates, covering threshold to near failure, at four R-ratios between 0.9 and 0.1 , were supplied by Airbus/UK.

Figure 16: Crack growth prediction in a complex helicopter component under spectrum loading

The analysis procedure is summarized as follows (Figure 17):

\section{Figure 17: Illustration of X-FEM model preparation.}

1. In the solid domain, identify the region where crack may penetrate; then specify the region using the X-FEM element type U1381. Use static analysis procedure in Abaqus and specify the time increment size as the maximum crack growth size. Apply the static load using nominal $\Delta P$.

2. Create the initial crack geometry and discretize the geometry using a triangular shell mesh. Assemble the crack mesh so that the intersection of the crack mesh and the solid mesh defines the initial, 1/4-circular edge crack at the corner of the raised hole.

3. Create the X-FEM specification file that associates the solid model file, the crack mesh file, and specifies X-FEM and level set representation parameters (most important parameters including: the enrichment zone size, the CTOD offset distance, and the level set representation grid size).

4. Execute the Abaqus analysis using the X-FEM user subroutine library.

5. The analysis results include regular solutions (displacement and stress variables) that are viewable in $\mathrm{CAE}$, the location of sampling tip points along the crack front at the end of each time increment, and the Ks solutions at those tip points.

6. Import the tip location and $K$ solution $(K(a)$ curve or converted beta curve) into AFGROW, along with the material fatigue data, for the life prediction.

The von Misses stress distribution for the component at the initial configuration is shown in Figure 18 , where the maximum far-field stress at the raised hole is $130 \mathrm{MPa}$. As can be seen from Figure 18, the stress field is intensified at the crack tip region.

Figure 18: X-FEM model and von Mises stress prediction at initial configuration. 
The deformed shape and the Von Misses stress distribution at the final configuration of fatigue analysis is shown in Figure 19.

Figure 19: X-FEM model and von Mises stress prediction at final configuration.

The crack profiles using two different mesh designs are showing in Figure 20. Note that the maximum growth size, $\Delta a_{\max }$ as in Eqn (27) is set to be $0.5 \mathrm{~mm}$.

Figure 20: Crack profiles comparison using coarse and fine mesh models.

After the determination of the functional relation between the crack size and its stress intensity factors along the crack front, a life prediction analysis is performed next using AFGROW. The spectrum to be used is the standard spectrum ASTERIX provided by Airbus/UK. ASTERIX has been derived from real strain data measured on a helicopter lift frame, using the same procedures as were used for the derivation of the rotor blade spectra HELIX and FEL. All three spectra are from the same 140 sorties representing 190.5 hours of flight. The sequence of maneuvers in each sortie is fixed. The total number of cycles in its complete form is $3.67 \times 10^{5}$ cycles. Given the fatigue test data at several applied ratios, a general curve-fit model is developed to characterize the load ratio $(\mathrm{R})$ dependent crack growth. The accuracy of the load ratio dependent fatigue model at several distinct R values is shown in Figure 21.

Figure 21: Load ratio dependent fatigue model at several distinct $r$ values.

The total analysis consists of 40 increments $(0.5 \mathrm{~mm}$ max. crack growth per increment $)$ in a single step, and took 3410 wall o'clock seconds on a laptop PC. A relatively coarse mesh (element size $0.3 \sim 1.0 \mathrm{~mm}$ ) generates very consistent crack profiles and $\mathrm{K}$ estimates by comparison with a finer mesh model. The crack travels to the threshold zone (about $5 \mathrm{~mm}$ of the $\mathrm{Y}$ coordinate) at about 390,000 cycles and before the end of the main plate $(\mathrm{Y}=17 \mathrm{~mm})$ at about 480,000 cycles. The early phase of predicted life is very different from the previous $2 \mathrm{D}$ predictions, which verifies the necessity of using 3D analysis. The early development of crack profiles, from the 1/4-circular initial crack to the straight cut-through crack in the main plate, significantly affects the fatigue life prediction result.

In X-FEM, the displacement enrichment using four branch functions allows a quite accurate estimate of the near-tip opening displacements and K's; therefore a relatively coarse and uniform mesh can be used in crack growth simulation. Using the narrow-band level set updates minimizes the mesh sensitivity of crack growth prediction. The current X-FEM solver can already predict fatigue life for constant-magnitude cyclic load case. For a variable-magnitude load case, fatigue life prediction is relied on a step-by-step, one-way coupling to AFGROW. In each step, a simplified crack growth model is adopted with the $K(a)$ profile at the free edge as the input data. 


\section{Conclusions}

A three-dimensional finite element method for the analysis of fatigue crack growth has been developed based on the extended finite element method (X-FEM). Both step function enrichments and singular enrichments to capture the behavior near the crack front are included. The resulting formulation can deal with small scale plasticity effectively. The crack morphology is described by level set methods that, in conjunction with X-FEM, allow for convenient modeling of growing cracks without remeshing: the crack geometry can be completely independent of the structure of the mesh. The method employs a new variant of the fast marching method which enables efficient updates of the level sets, and thus the crack geometry. In this method, triquintic interpolants are used for front reconstruction and the level set updates that are used to model crack growth. Methods for dealing with crack closure effects have also been described.

The methodologies have been incorporated in the Abaqus program. The user element option is employed to implement the X-FEM elements. By means of the UXTERNALDB the analysis flow is controlled, including the integration of the fast marching method solver and the special output features for $\mathrm{X}$-FEM. Verification and validation studies were performed using standard and modified compact tension specimens.

For the standard CT specimen, the fracture parameters predicted from the X-FEM toolkit agree very well with the analytical solution. A parametric study has also been performed to explore the mesh sensitivity and the perturbation of the XFEM modeling parameters on the prediction of fracture parameter, crack path, and fatigue life. Using the modified CT specimens for miss-hole and sink-hole configurations given by Miranda et al. (2003), distinct fatigue crack growth behavior was simulated and showed to have an excellent agreement with the experimental observations. The X-FEM prediction of the fatigue life also agrees well with the reported test data.

Finally, the full capability of the tool was revealed via its application to a 3D helicopter lift frame subjected to a real spectrum loading. Excellent agreement has been achieved by comparing the model prediction with the test data provided by Airbus at two fatigue crack growth stages. The 3D X-FEM analysis has also demonstrated that the use of a 2D through-the-thickness crack would be too conservative since a significant number of cycles have been exhausted in forming the 2D crack from an initial 3D crack.

\section{Acknowledgements}

The authors gratefully acknowledge the support for X-FEM solid element and life prediction development from ONR 331 under contract N00014-07-C-0442, N00014-08-C-0433, and N00014-09-C-0416 with Dr. Paul Hess as the program manager. Some X-FEM framework development was also supported by AFRL/MLBC under contract FA8650-07-C-5015 with Dr. David Mollenhauer as 
the program manager. We also very appreciate Dr. Jianhong Lin of Airbus/UK for providing data and valuable advices on the demonstration example problem.

\section{References}

[1] Abdelaziz Y, Hamouine A. A survey of the extended finite element. Comp Struct. 86:1141-1151. 2008.

[2] Belytschko T, Lu Y, Gu L. Element-free Galerkin methods. Int J Num Meth Eng. 37:229-56, 1994.

[3] Belytschko T, Krongauz Y, Organ D, Fleming M, Krysl P. Meshless methods: an overview and recent developments. Comp Meth Appl Mech Eng. 139:3-47, 1996.

[4] Belytschko T, Black T. Elastic crack growth in finite elements with minimal remeshing. Int J Num Meth Eng. 45:601-20, 1999.

[5] Belytschko T, Gracie R, Ventura G. A review of extended/generalized finite element methods for material modeling. Model Simu Mat Sci Eng. 17(4): , 2009.

[6] Bordas S, Moran B. Enriched finite elements and level sets for damage tolerance assessment of complex structures. Eng Fract Mech. 73:1176-201, 2006.

[7] Carter, BJ, Wawrzynek PA, Ingraffea AR. Automated 3-D crack growth simulation. Int J Numer Meth Eng. 47:229-253, 2000.

[8] Chopp DL. Some improvements of the fast marching method. SIAM J Sci Comp. 23(1):230-244, 2001.

[9] Chopp DL, Sukumar N. Fatigue crack propagation of multiple coplanar cracks with the coupled extended finite element/fast marching method. Int J Eng Sci. 41:845-69, 2003.

[10] Cruse T. Boundary element analysis in computational fracture mechanics. Kluwer-Dordrecht. 1988.

[11] Daux C, Moes N, Dolbow J, Sukumar N, Belytschko T. Arbitrary branched and intersecting cracks with the extended finite element method. Int J Num Meth Eng. 48:1741-60, 2000.

[12] Dolbow J. An extended finite element method with discontinuous enrichment for applied mechanics. PhD thesis, Northwestern University. 1999.

[13]Dolbow J, Moes N, Belytschko T. An extended finite element method for modeling crack growth with frictional contact. Comput Meth Appl Mech Eng. 190:6825-46, 2001.

[14]Dolbow J, Devan A. Enrichment of enhanced assumed strain approximations for representing strong discontinuities: addressing volumetric incompressibility and the discontinuous patch test. Int J Num Meth Eng. 59:47-67, 2004.

[15]Elguedj T, Gravouil A, Combescure A. Appropriate extended functions for XFEM simulation of plastic fracture mechanics. Comp Meth App Mech Eng. 195:501-515, 2006.

[16]Giner E, Sukumar N, Tarancon JE, Fuenmayor FJ. An Abaqus implementation of the extended finite element method. Eng Fract Mech. 76:347-368, 2009.

[17] Gravouil A, Moes N, Belytschko T. Non-planar 3D crack growth by the extended finite element and level sets part II: level set update. Int J Num Meth Eng. 53:2569-86, 2002.

[18] Harter J. AFGROW Users Guide and Technical Manual. AFRL-VA-WP-TR-2008-XXXX, Air Force Research Laboratory, July 2008. 
[19]Henshell RD, Shaw KG. Crack tip finite elements are unnecessary. Int J Numer Meth Eng. 9:495-507, 1975.

[20]Huang R, Sukumar N, Pre'vost J. Modeling quasi-static crack growth with the extended finite element method part II: numerical applications. Int J Solids Struct 40:7539-52, 2003.

[21] Karihaloo BL, Xiao QZ. Modeling of stationary and growing cracks in FE framework without remeshing: a state-of-the-art review. Comp Struct. 81:119-29, 2003.

[22] Khoei AR, Nikbakht M. Contact friction modeling with the extended finite element method (X-FEM). J Mat Proces Tech, (177)1-3:58-62, 2006.

[23] Khoei AR, Biabanaki SOR, Anahid M. A Lagrangian-extended finite element method in modeling large-plasticity deformations and contact problems, Int J Mech Sci, (51)5:384-401. 2009.

[24]Lei Y. Finite element crack closure analysis of a compact tension specimen, Int J Fat. 30: 21-31, 2008.

[25]Lua J, Shi J, Liu P, Collette M. Curvilinear crack growth and remaining life prediction of aluminum weldment using X-FEM, 49th AIAA/ASME/ASCE/AHS/ASC Structures, Structural Dynamics, and Materials Conference. \#AIAA-2008-1839, 2008.

[26]Lua J, Englestad, S. Pi-Joint reliability assessment using X-FEM/script, 50th AIAA/ASME/ASCE/AHS/ASC Structures, Structural Dynamics, and Materials Conference. 2009.

[27] Maligno AR, Rajaratnam S, Leen SB, Williams EJ. A three-dimensional (3D) numerical study of fatigue crack growth using remeshing techniques. Eng Fract Mech. 77:94-111, 2010.

[28] Miranda ACO, Meggiolaro MA, Castro JTP, Matha LF, Bittencourt TN. Fatigue life and crack path predictions in generic 2D structural components. Eng Fract Mech. 70:1259-1279, 2003.

[29] Melenk JM, Babuska I. The partition of unity finite element method: basic theory and applications. Comp Meth Appl Mech Eng 139:289-314, 1996.

[30] Moes N, Dolbow J, Belytschko T. A finite element method for crack growth without remeshing. Int J Num Meth Eng. 46:131-50, 1999.

[31] Moes N, Gravouil A, Belytschko T. Non-planar 3D crack growth by the extended finite element and level sets part I: mechanical model. Int J Num Meth Eng. 53:2549-68, 2002.

[32] Nagtegaal J C, Parks DM, Rice JR. On numerically accurate finite element solutions in the fully plastic range. Comp Meth Applied Mech Eng. (4): 153-177, 1974.

[33] Nistro I, Pantale' O, Caperaa S. On the modeling of the dynamic crack propagation by extended finite element method: numerical implantation in DYNELA code. In: VIII international conference on computational plasticity, COMPLAS VIII, Barcelona; 2005.

[34] Newman JCR, Irving, PE, Lin J, Le DD. Crack growth predictions in a complex helicopter component under spectrum loading, Fat Fract Eng Mat Struct. 29:949-958, 2006.

[35] Oden TJ, Belytschko T, Babuska, Hughes TJR, Research directions in computational mechanics. Comp Meth Applied Mech Eng. 192: 913-922, 2003.

[36] Osher S, Sethian J. Fronts propagating with curvature dependent speed: algorithms based on Hamilton-Jacobi formulations. J Comp Phys. 79(1):12-49, 1988.

[37] Pantale' O, Caperaa S, Rakotomalala R. Development of an object oriented finite element program: application to metal forming and impact simulation. J-CAM. 186(1-2):341-51, 2004.

[38] Pre'vost J. Dynaflow. Princeton University. 1983. 
[39] Sethian J. Fast marching methods. SIAM Rev. 41(2):199-235, 1999.

[40] Shi J, Lua J, Waisman H, Belytschko T, Sukumar N. X-FEM toolkit for automated crack onset and growth prediction, 49th AIAA/ASME/ASCE/AHS/ASC Structures, Structural Dynamics, and Materials Conference. \#AIAA-2008-1763, 2008.

[41] Stolarska M, Chopp DL, Moes N, Belyschko T. Modeling crack growth by level sets in the extended finite element method. Int J Num Meth Eng. 51:943-60, 2001.

[42] Sukumar N, Chopp DL, Moran B. Extended finite element method and fast marching method for three-dimensional fatigue crack propagation. Eng Fract Mech. 70:29-48, 1999.

[43] Sukumar N, Moes N, Moran B, Belytschko T. Extended finite element method for three-dimensional crack modelling. Int J Num Meth Eng, 48:1549-70, 2000.

[44] Sukumar N, Pre'vost JH. Modeling quasi-static crack growth with the extended finite element method part I: computer implementation. Int J Solids Struct 40:7513-37, 2003.

[45] Sukumar N, Chopp DL, Bechet E, Moes N. Three-dimensional non-planar crack growth by a coupled extended finite element and fast marching method, Int J Num Meth Eng. 76(5):727-748, 2008.

[46] Ventura G. On elimination of quadrature subcells for discontinuous functions in the extended finite element method. Int J Num Meth Eng 66:761-95, 2006.

[47]Xiao QZ, Karihaloo BL. Improving the accuracy of X-FEM crack tip fields using higher order quadrature and statically admissible stress recovery. Int J Num Meth Eng 2006;66:1378-410.

[48]Zienkiewicz, O C, Zhu, J Z. The superconvergence patch recovery and a posteriori estimates. Part 1: The recovery technique, Int J Num Meth Eng. 33: 1331-1364, 1992.

[49]Zienkiewicz, OC, Taylor, RL. The Finite Element Method, Fifth edition, Butterworth-Heinemann. 2000. 


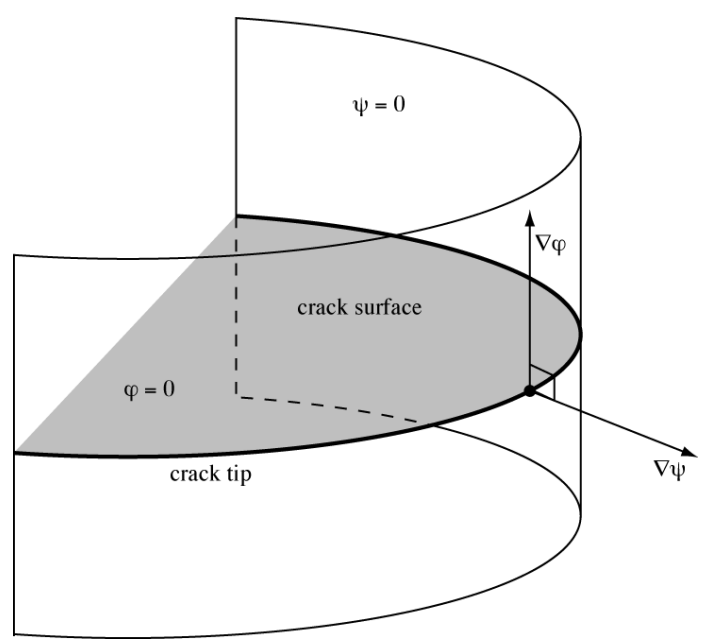

Figure 1: Illustration of the crack surface function $\varphi$ and the crack front function $\psi$.

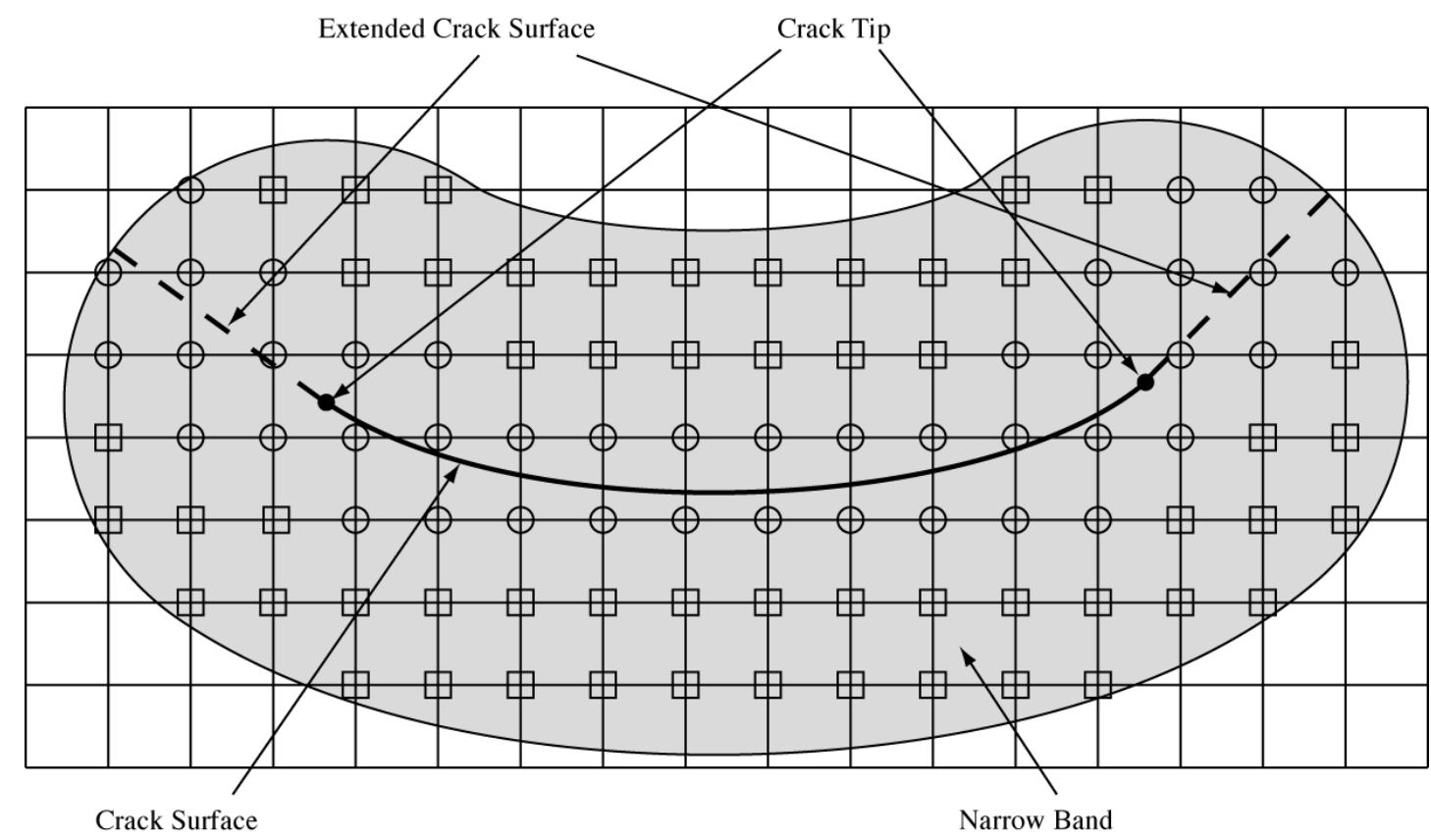

Figure 2: Illustration of the narrowband, in gray, of points around the crack surface, in cross-section, where the FMM mesh maintains signed values. The circled points are where the initialized values are computed directly and the squared points are where the values are computed using the FMM. 


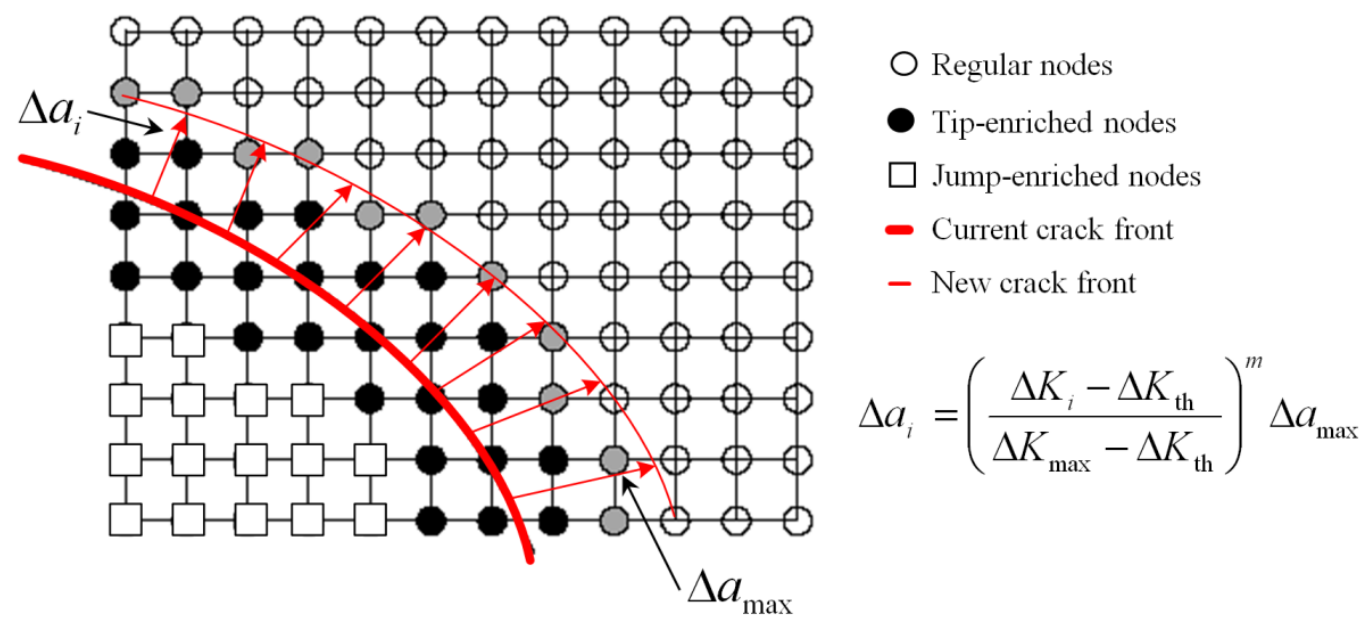

Figure 3: Overview of crack tracking and growth control.
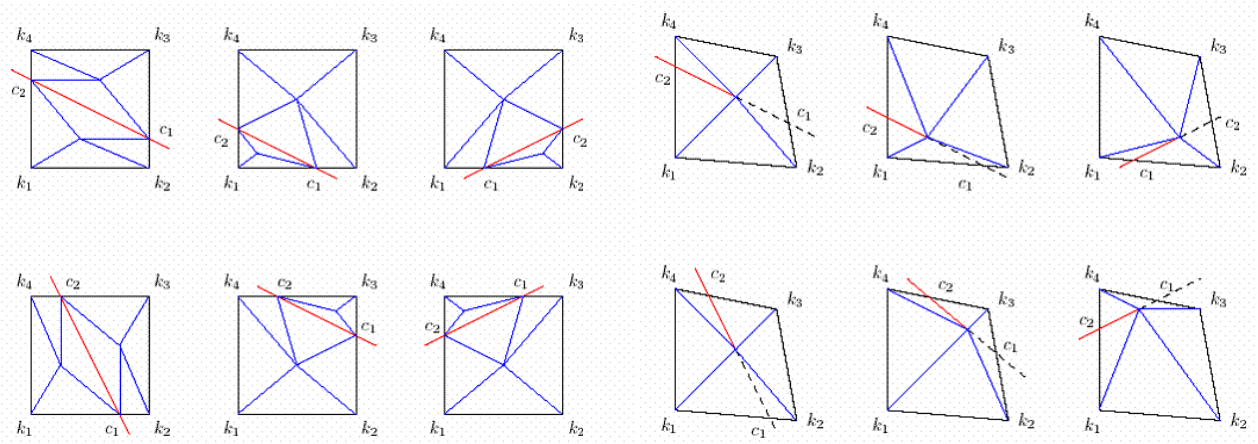

(a) (b)

Figure 4: Slicing scheme on the quad facet: (a) scenarios of facets being completely cut by a crack surface represented by the line $c_{1}-c_{2}$; and (b) scenarios of facets partially cut by a crack surface.

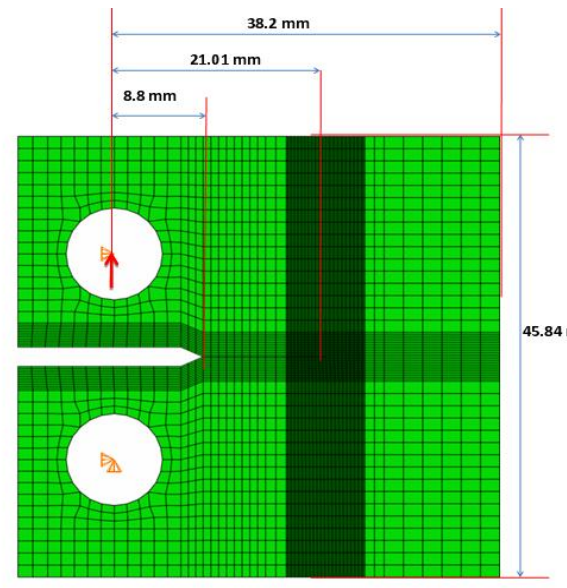

(a)

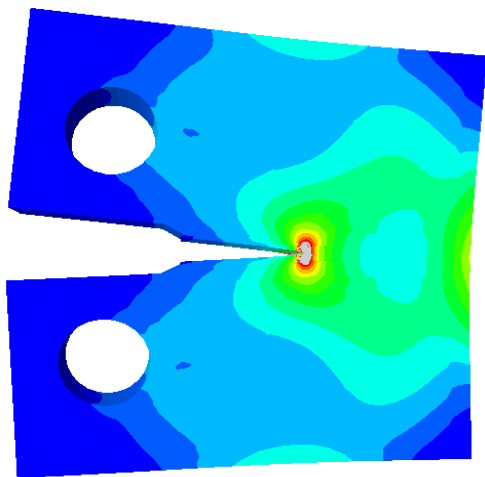

(b)

Figure 5: K validation using a standard compact test specimen. (a) Specimen dimensions and $\mathrm{X}$-FEM mesh design; (b) von Mises stresses in deformed shape. 


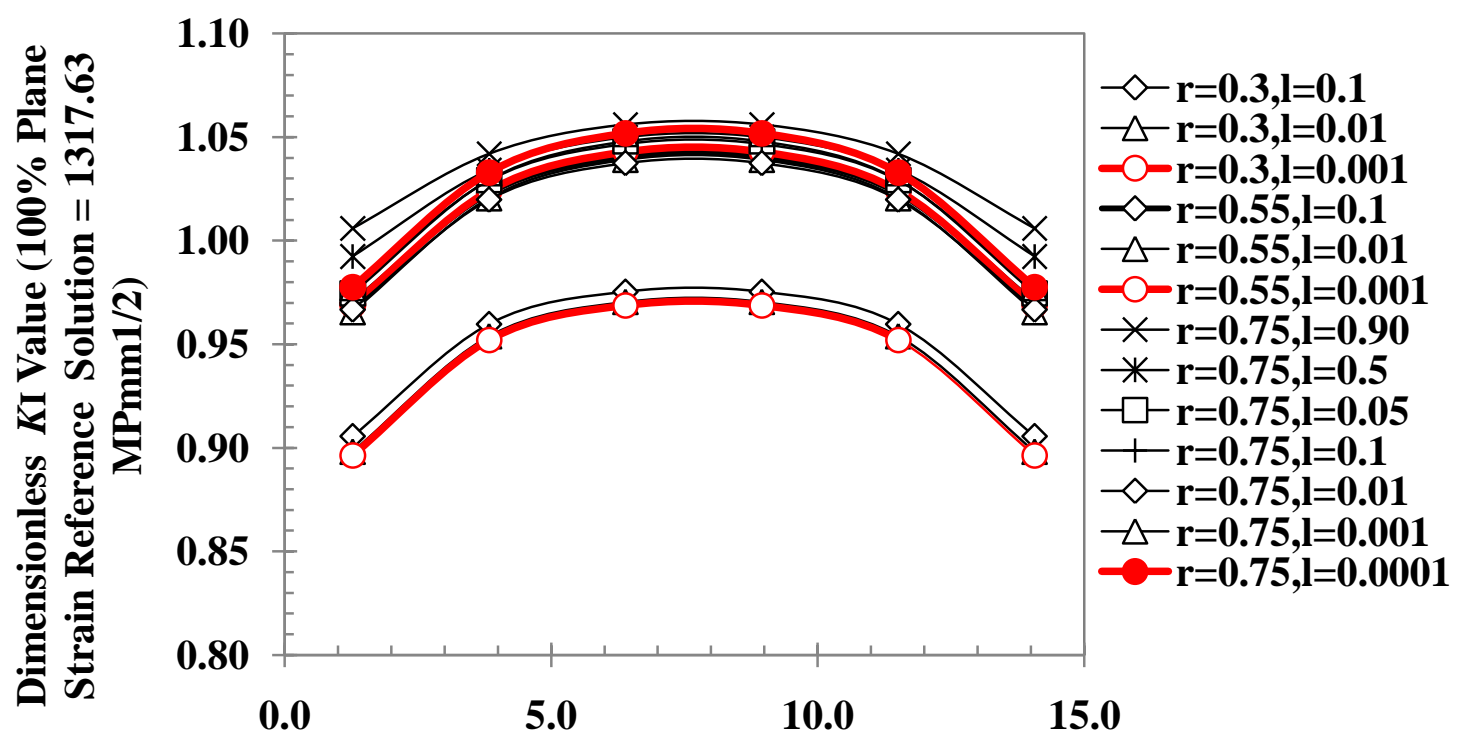

Tip Coordinate in Through-Thickness Direction (mm)

from Boundary to Boundary

(a)

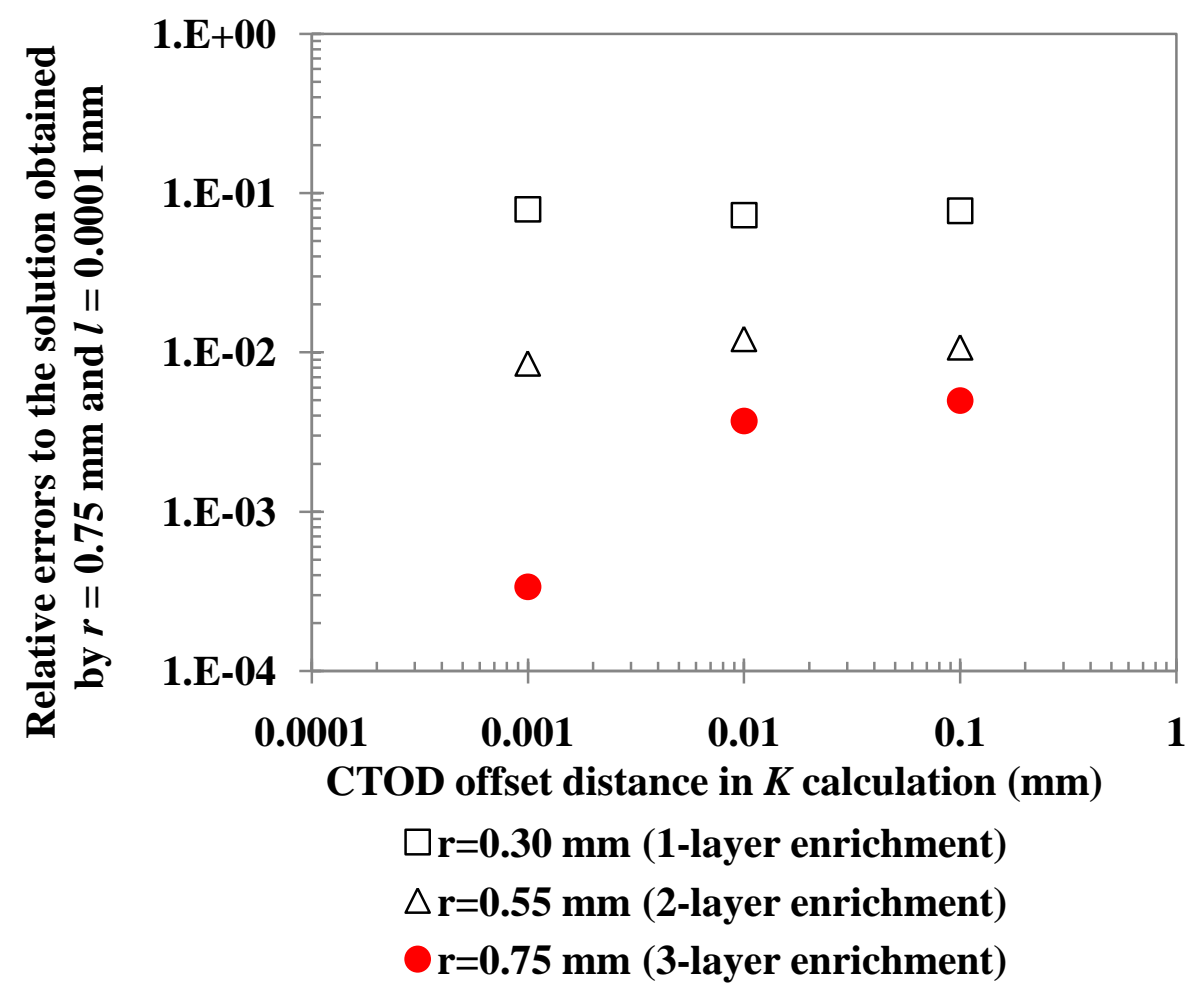

(b)

Figure 6: (a) Dimensionless $K$ values in reference to Eqn (47) using X-FEM with a combination of parameter settings; and (b) relative errors in $K$ prediction as a function of the tip enrichment radius and the CTOD offset distance. 


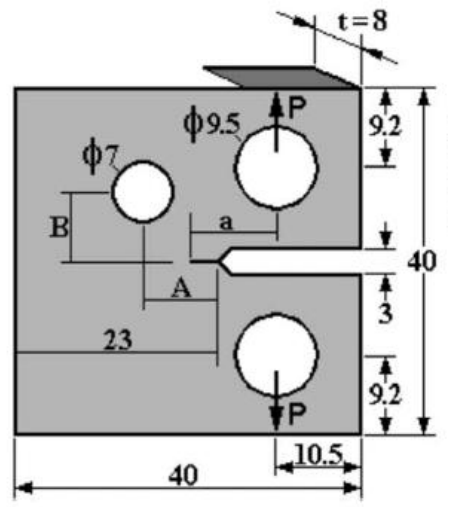

Figure 7: Geometry of the modified CT specimen. $A=8.3 \mathrm{~mm}$ and $B=8.1 \mathrm{~mm}$ for the miss-hole configuration; and $A=8.4 \mathrm{~mm}$ and $B=6.9 \mathrm{~mm}$ for the sink-hole configuration.
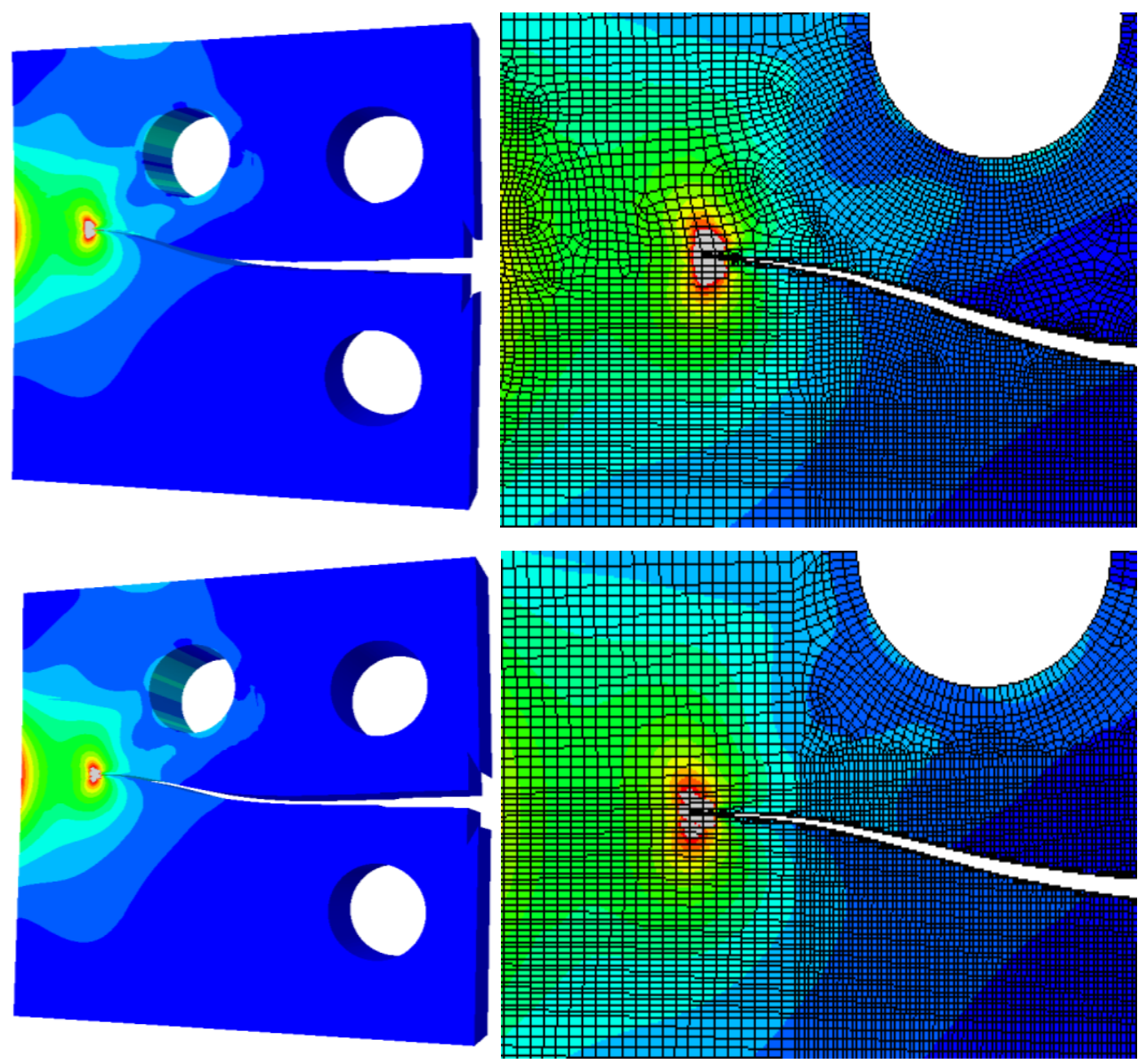

Figure 8: Mesh sensitivity study of crack growth patterns for the miss-hole CT specimen under constant cyclic loading 


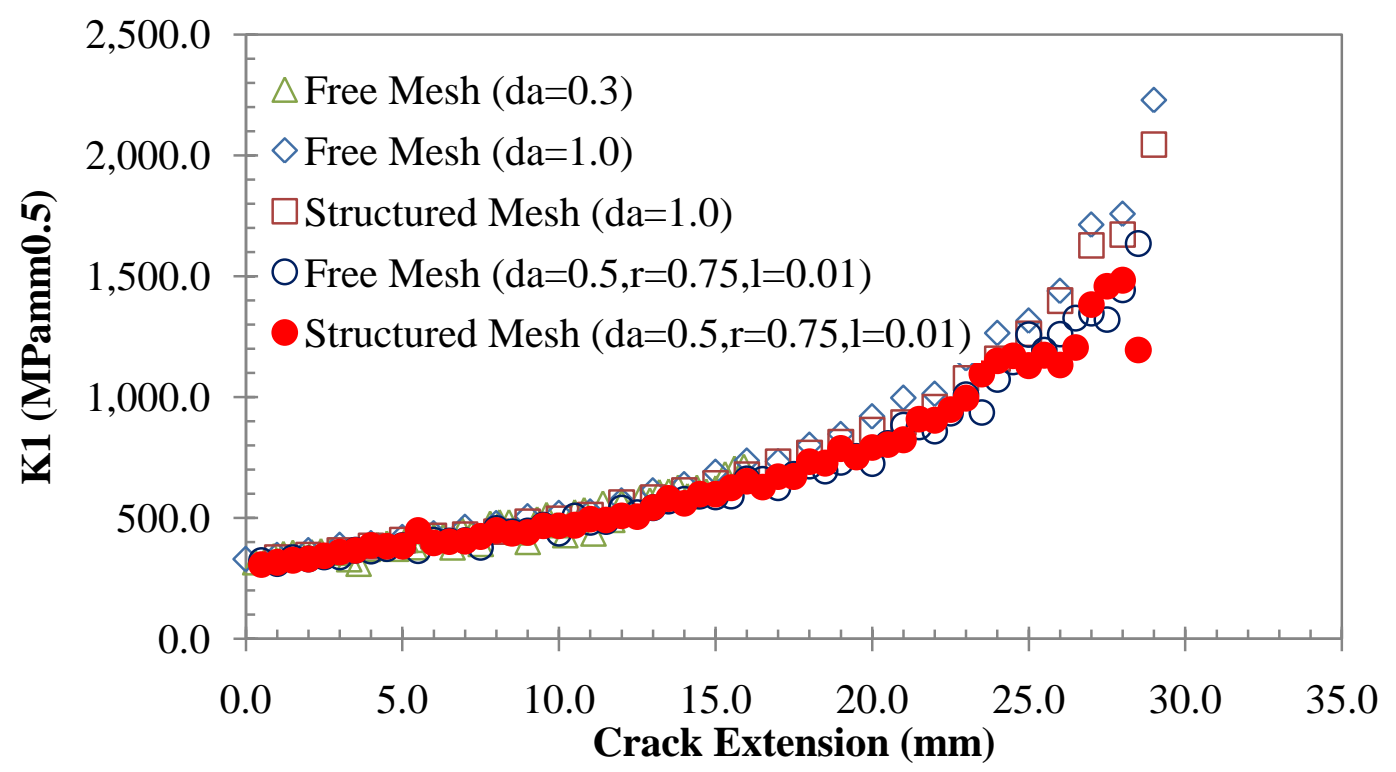

(a)

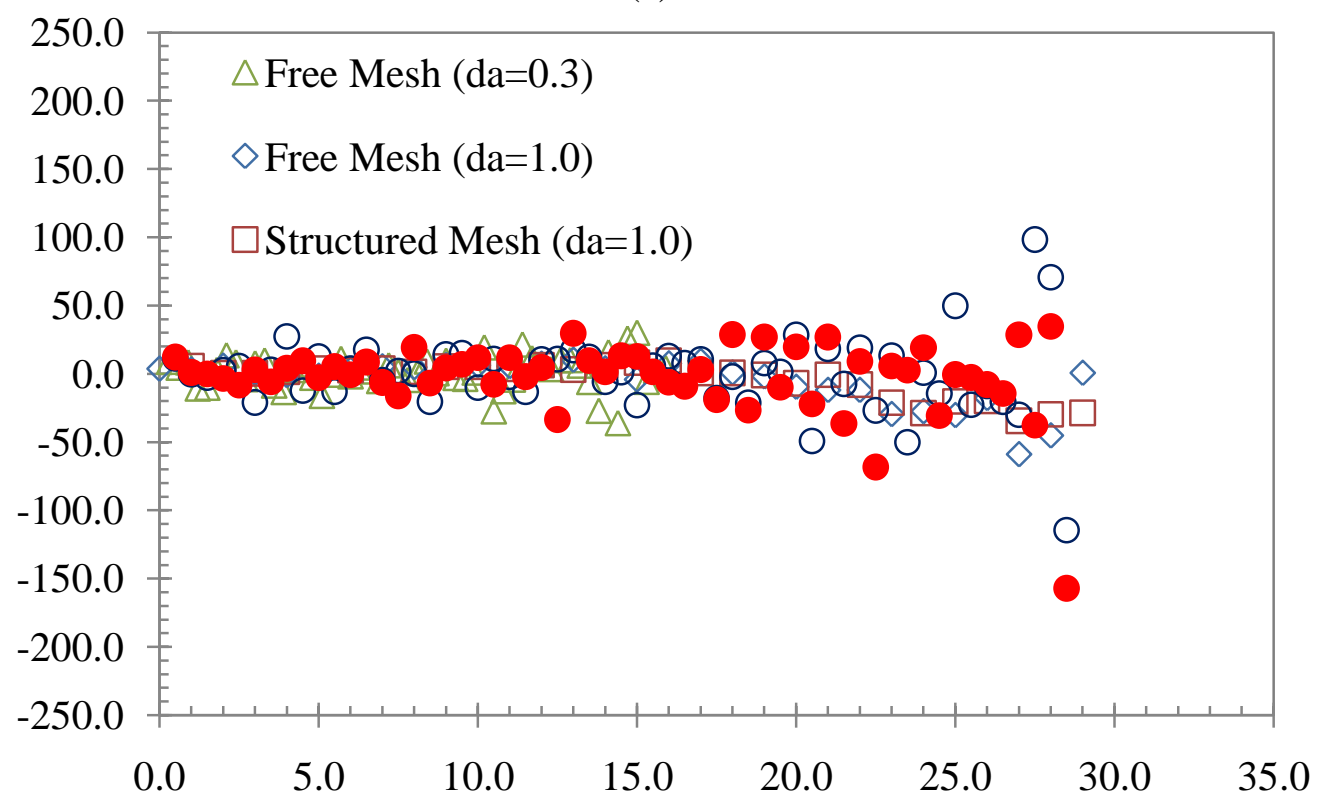

(b)

Figure 9: Stress intensity factors $K_{I}(a)$ and $K_{I I}$ (b) as functions of crack extension size, using combinations of $X-F E M$ analysis controlling parameters. 


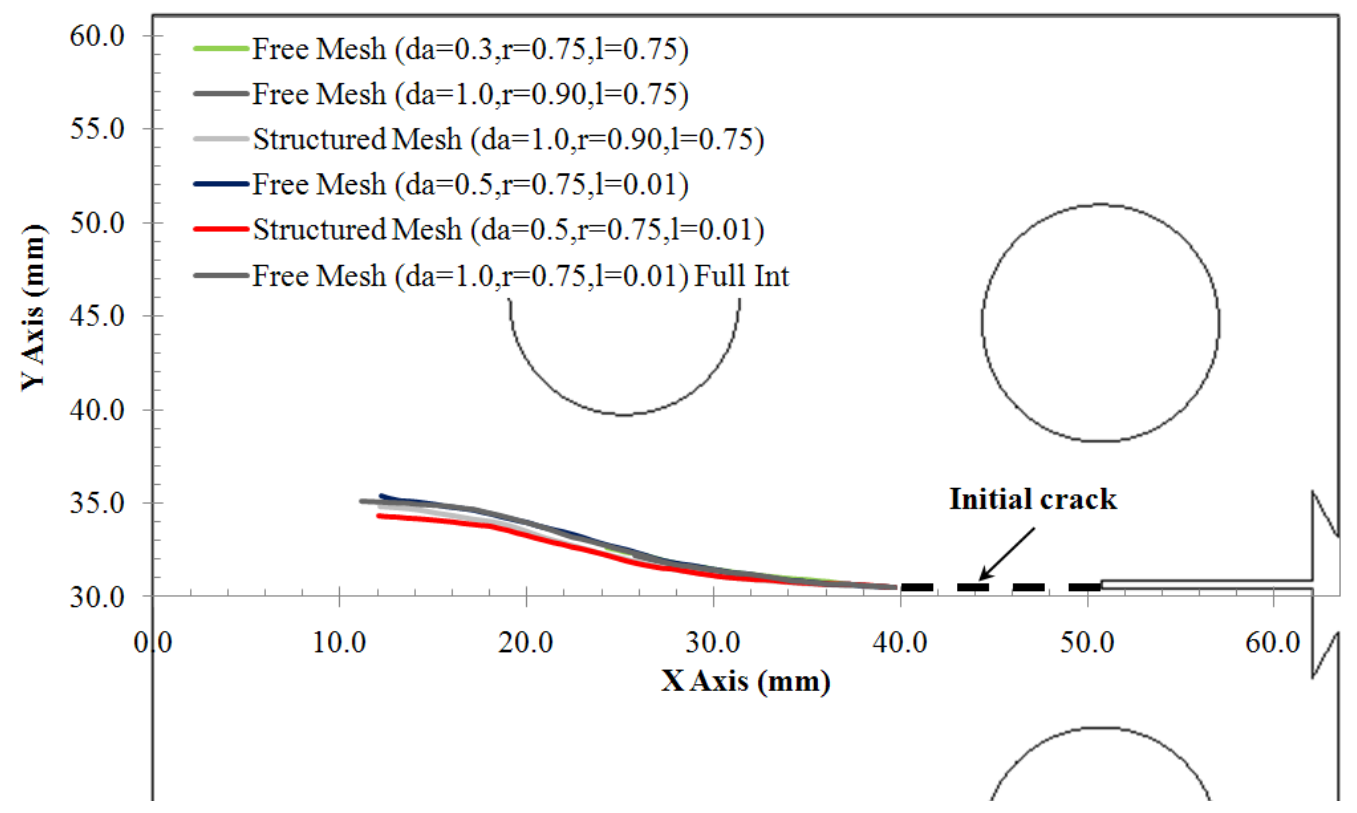

(a)

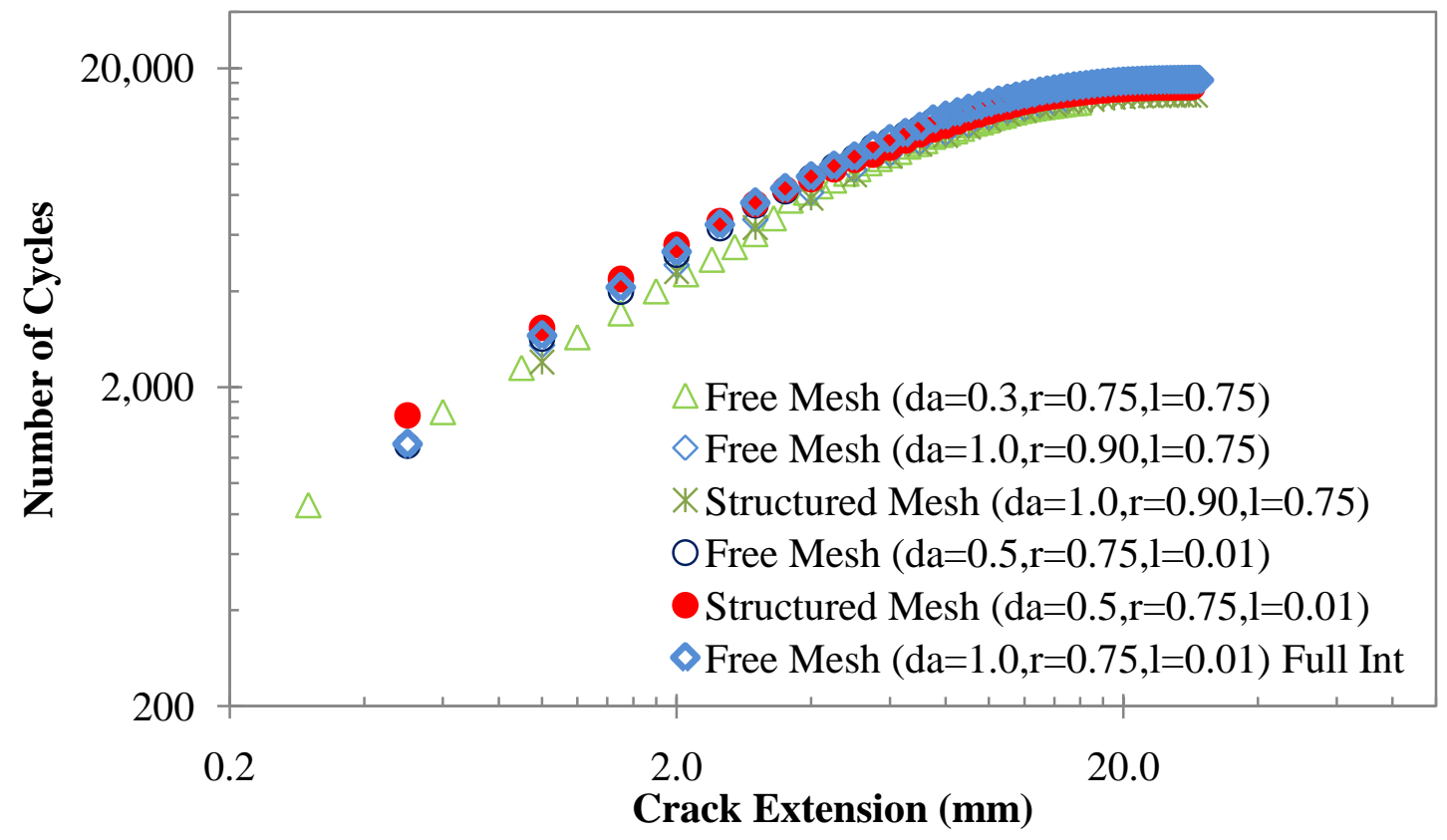

(b)

Figure 10: Crack profile (a) and fatigue life prediction (b) as functions of crack extension size, using combinations of $\mathrm{X}$-FEM analysis controlling parameters. 


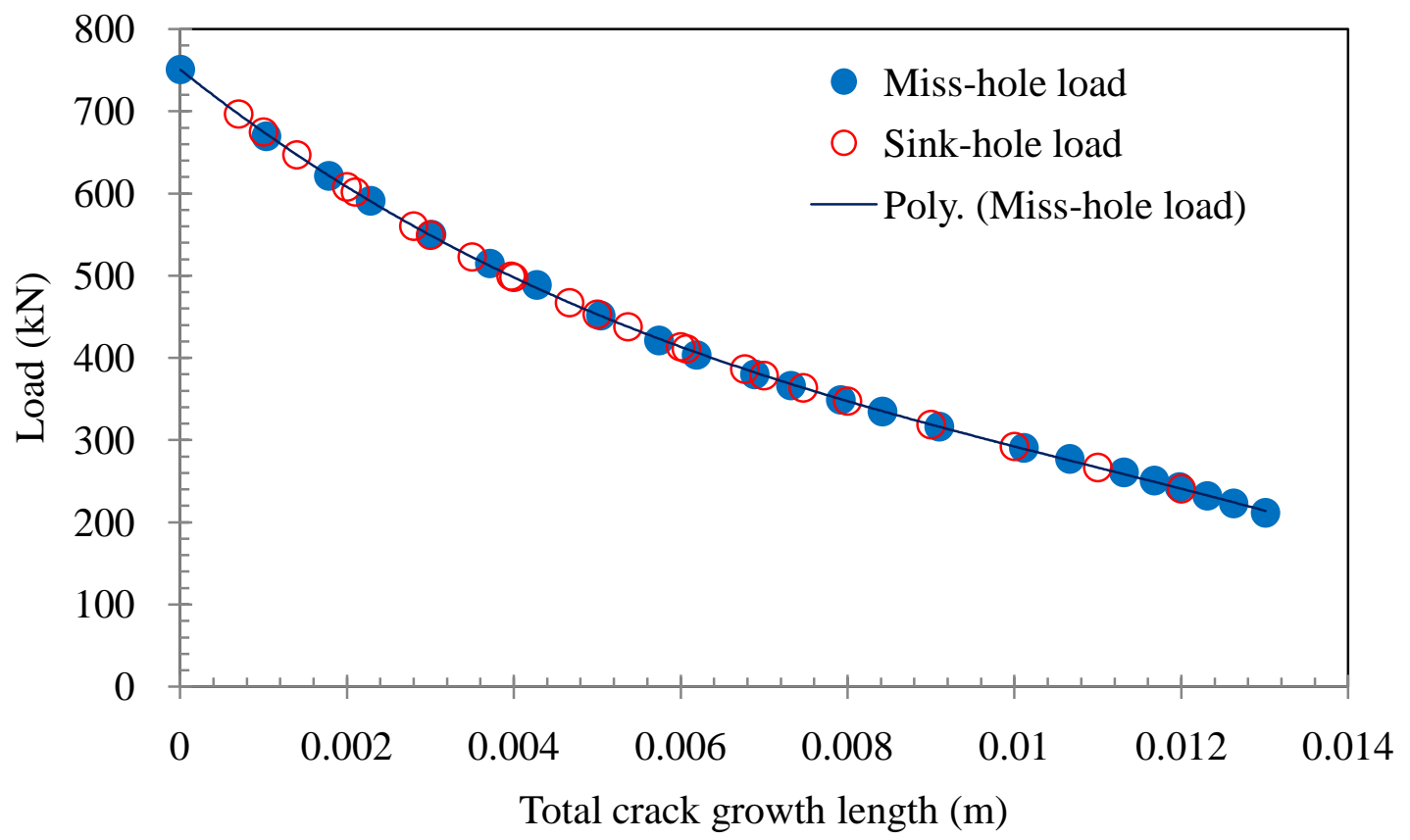

Figure 11: Loading history for the modified-hole CT specimen analyses.

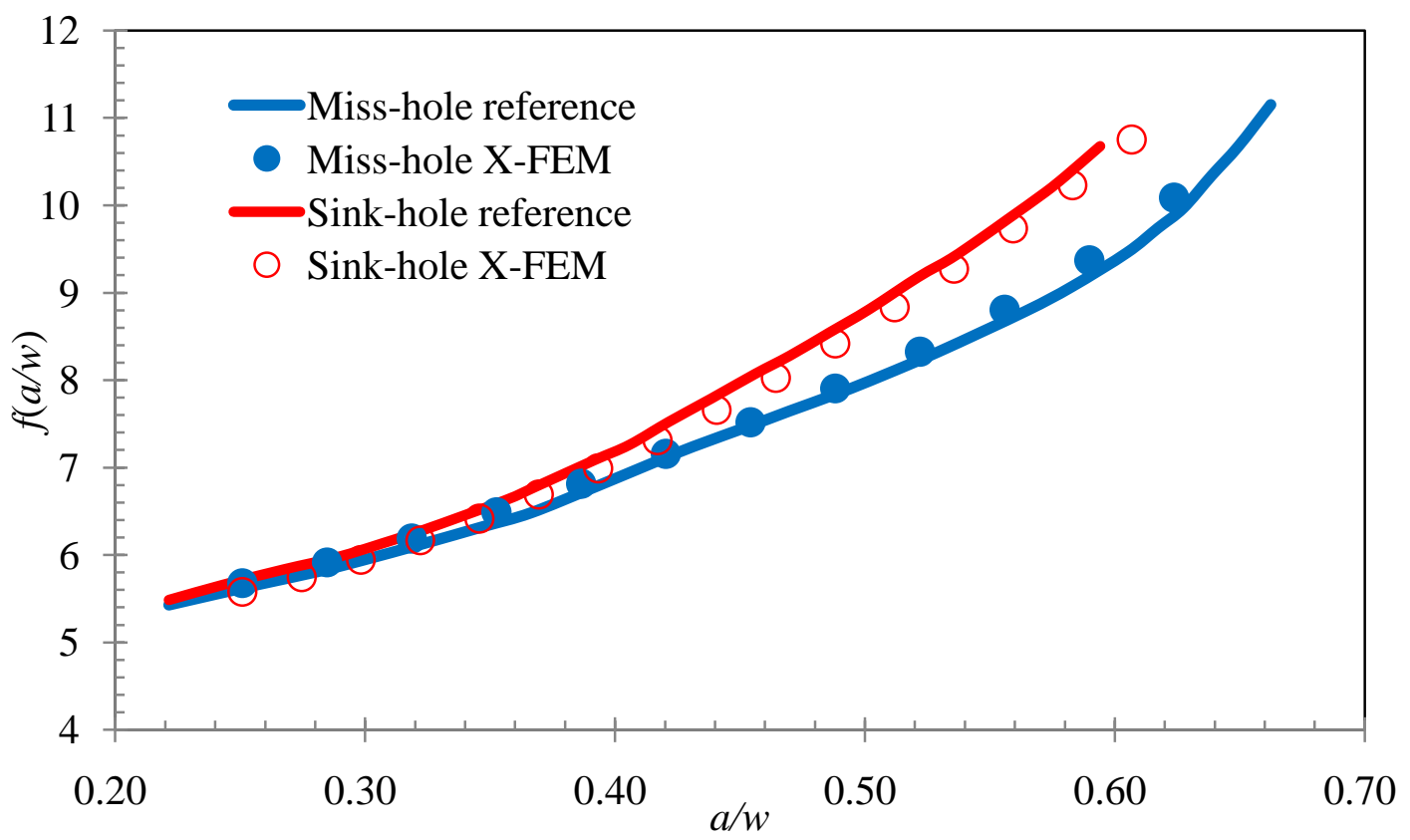

Figure 12: Comparison of calculated $f(a / w)$ for the modified-hole specimens. 


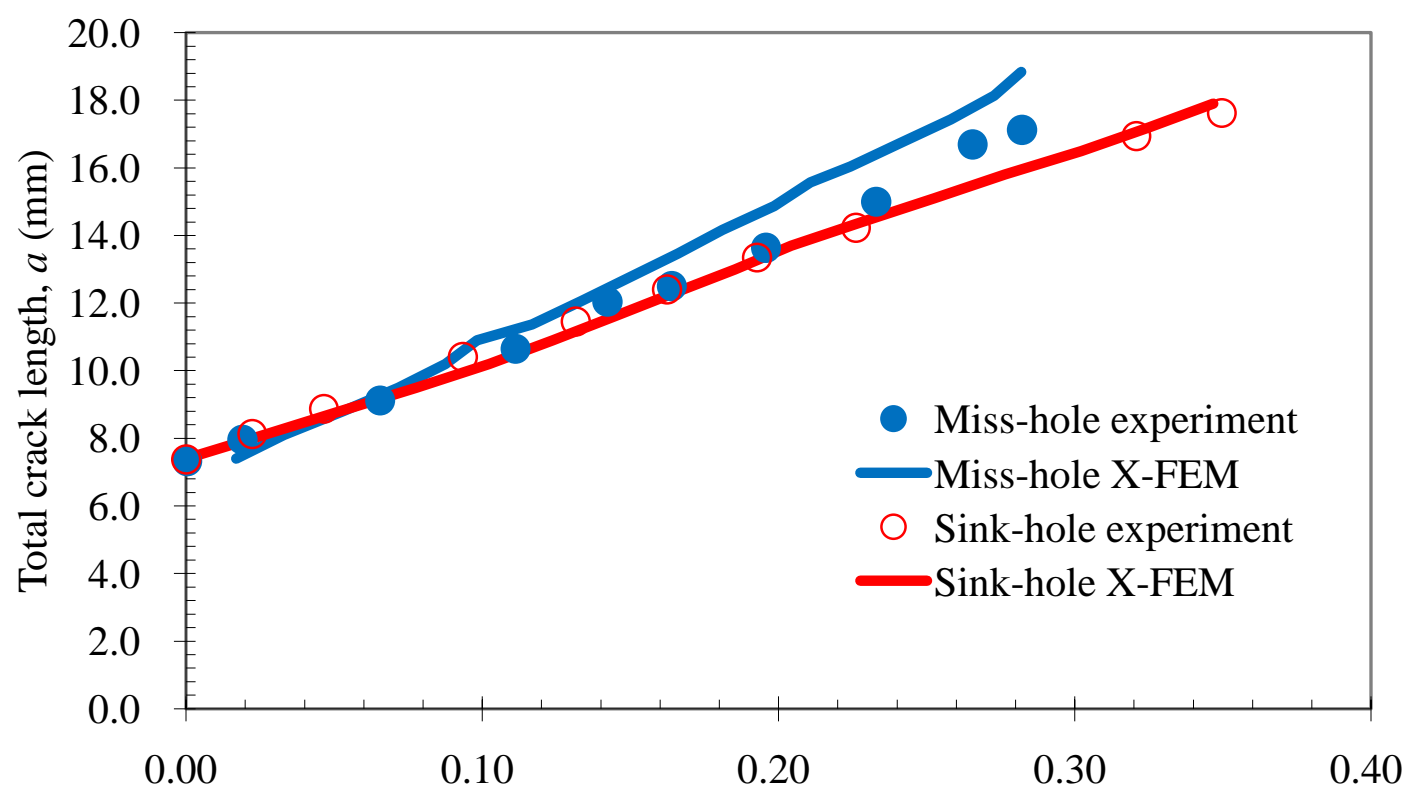

Fatigue cycles (in millions)

Figure 13: Comparison of fatigue life prediction for the modified-hole specimens
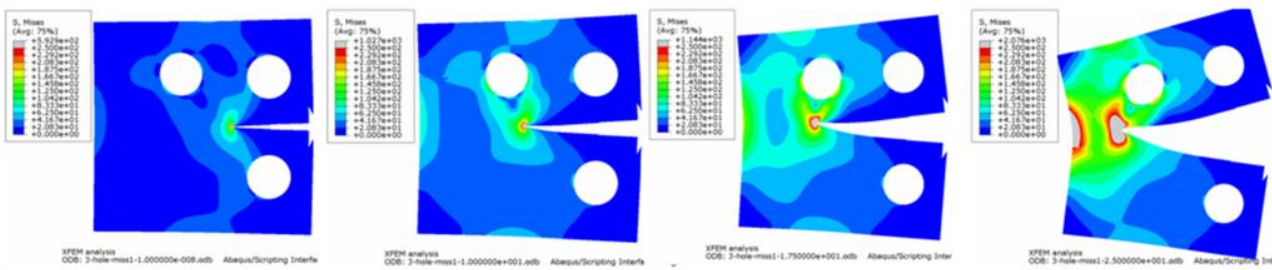

Miss-Hole Snapshots
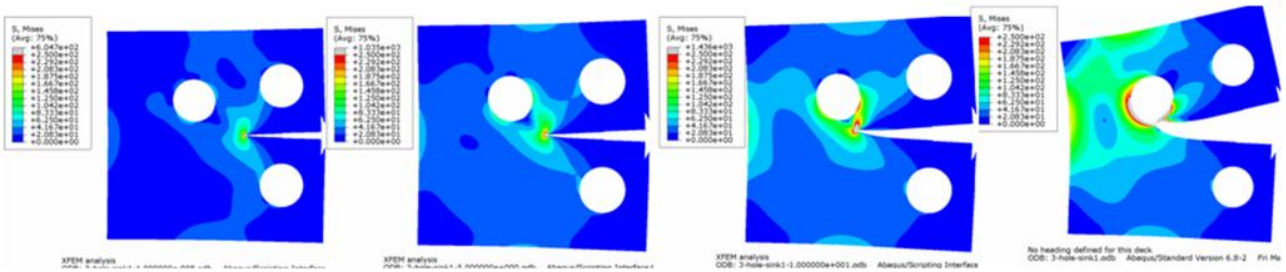

Sink-Hole Snapshots

Figure 14: Snapshots of CT specimen von Mises stress as crack grows. 

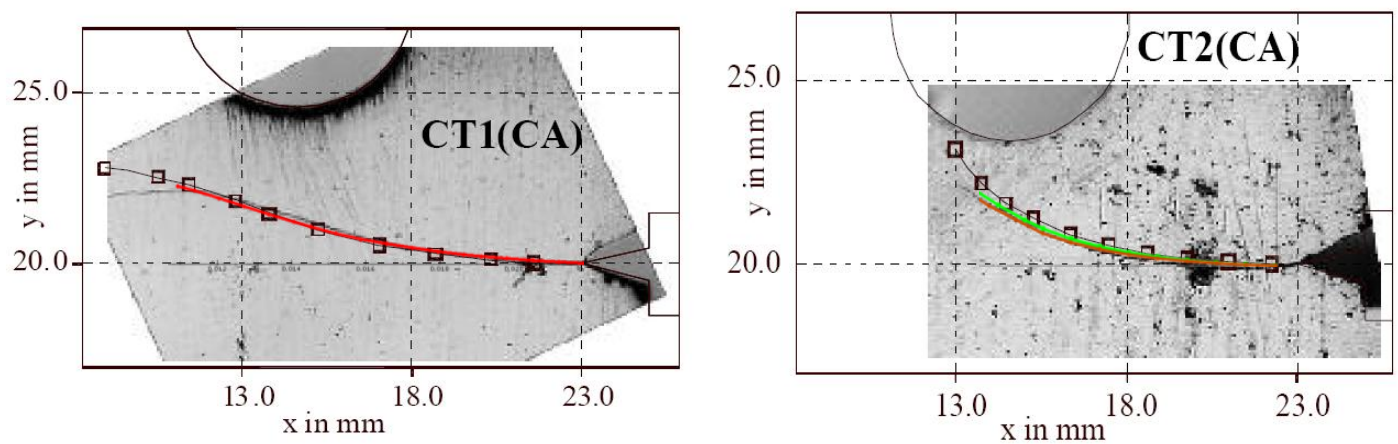

Figure 15: Comparison of crack path prediction: miss-hole (CT1) on the left and sink-hole (CT2) on the right.
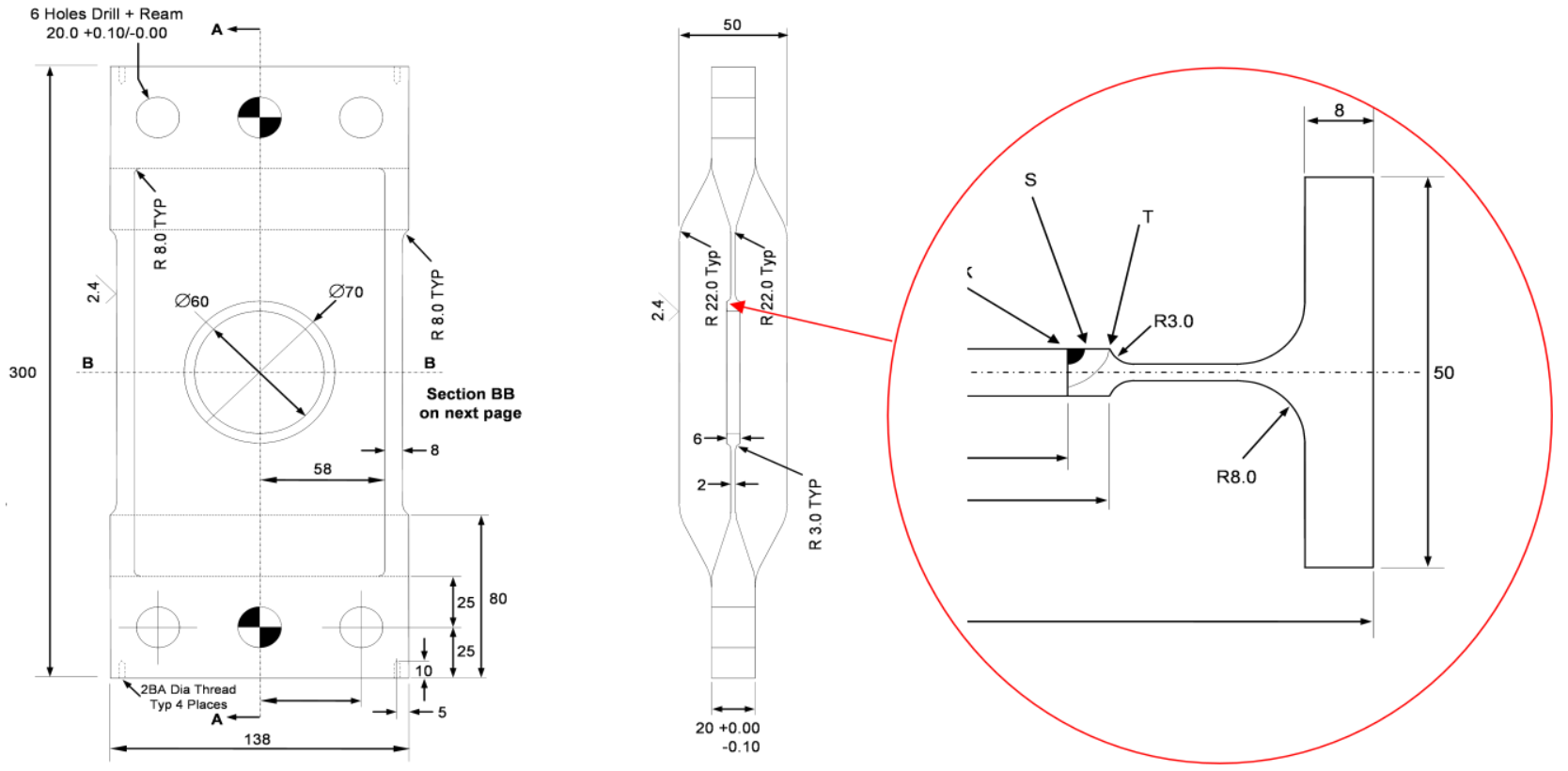

Figure 16: Crack growth prediction in a complex helicopter component under spectrum loading 


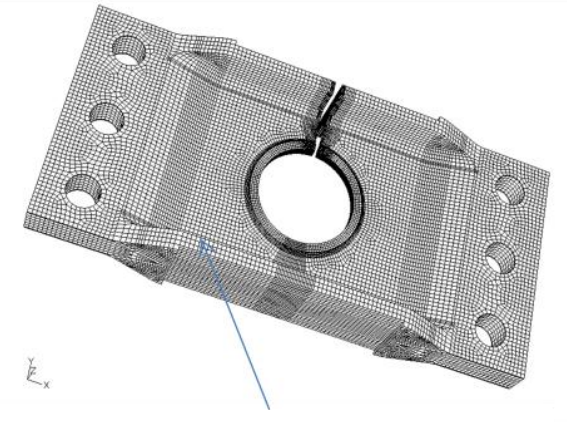

Solid Model excluding the

$\mathrm{X}$-FEM user element region

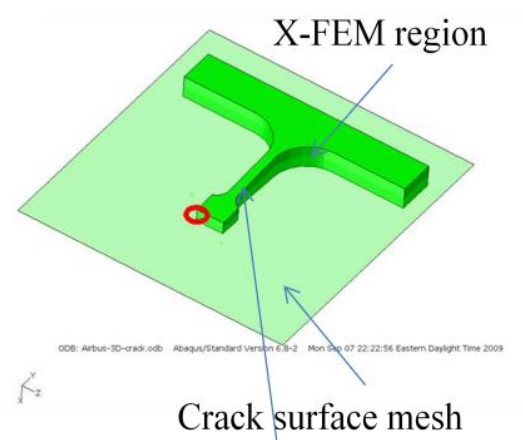

Crack front (highlighed)

Figure 17: Illustration of X-FEM model preparation.

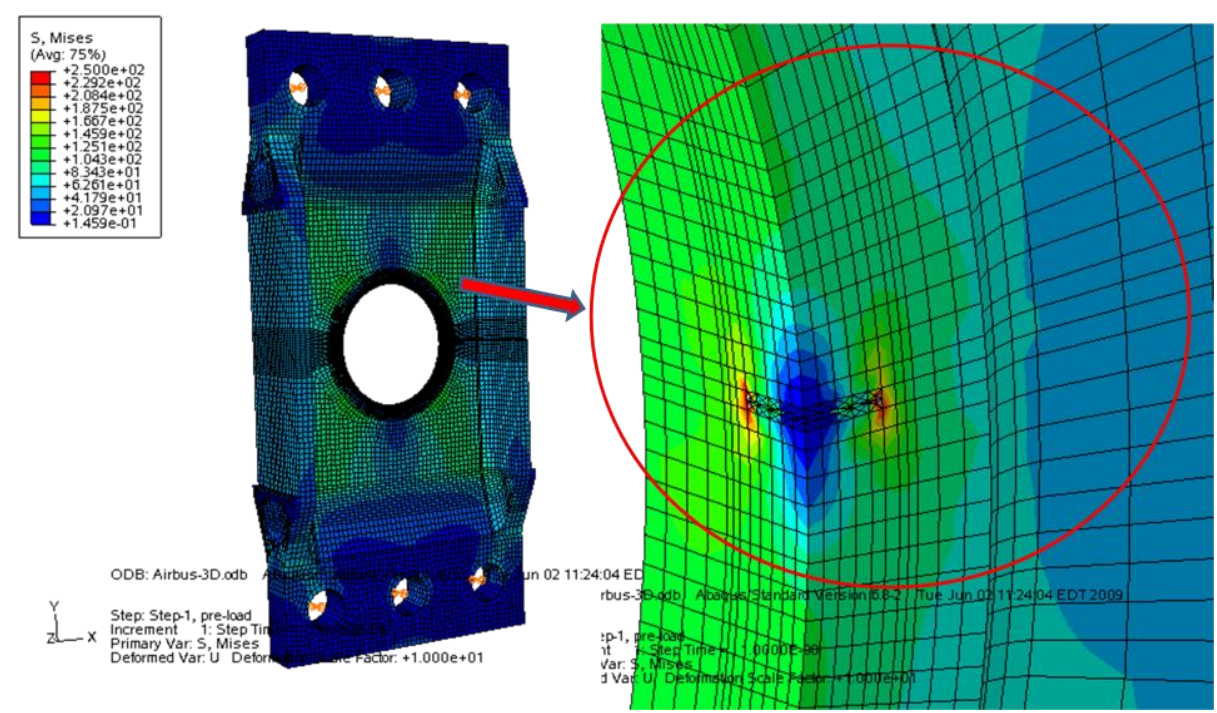

Figure 18: $X$-FEM model and von Mises stress prediction at initial configuration.

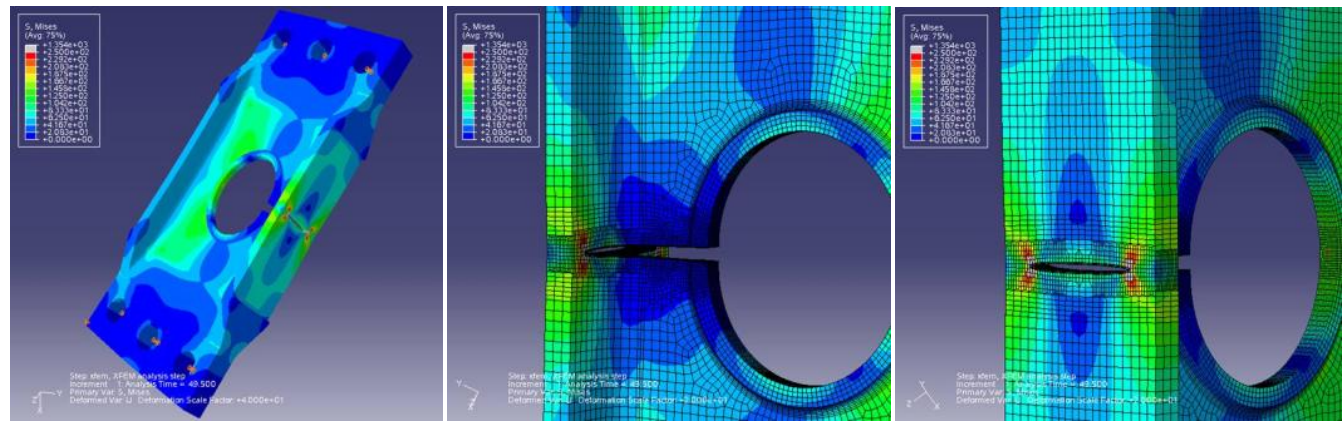

Figure 19: $X-F E M$ model and von Mises stress prediction at final configuration. 


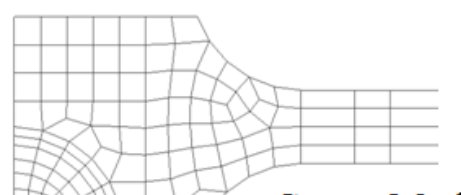

Coarse Mesh

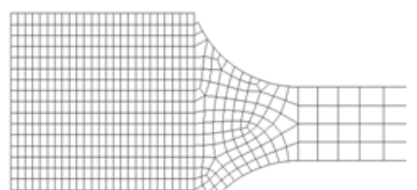

Fine Mesh

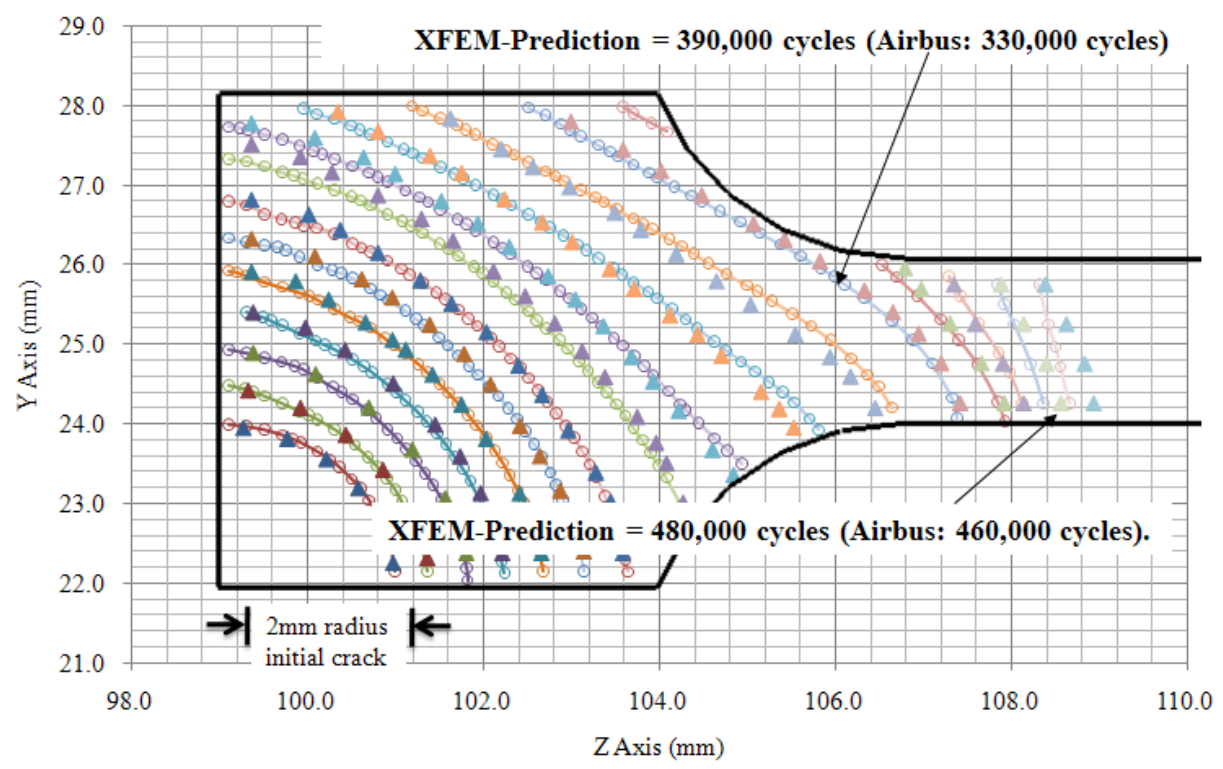

- Coarse $\mathrm{da}=0.0$

- Coarse da $=0.5$

- Coarse da $=1.0$

- Coarse da $=1.5$

- Coarse da $=2.0$

- Coarse $\mathrm{da}=2.5$

- Coarse da $=3.0$

Coarseda=4.0

- Coarse da $=4$.

Coarseda=s.

4 Coarse $\mathrm{da}=6$.

Coarse da $=7.0$

- Coarse da $=8.0$

Coarse da $=9.0$

$\Delta$ Coarse $\mathrm{da}=10.0$

Coarse da $=105$

Coarse $d a=110$

- Fine $\mathrm{d} a=0.0$

- Fine $\mathrm{da}=0.0$

- Fine $d a=0.5$

- Fine da=1.0

- Fine $\mathrm{da}=1.5$

- Fine $\mathrm{da}=2.0$

- - Fine $\mathrm{da}=2.5$

- - Fine $\mathrm{da}=3.0$

$-0-$ Fine $\mathrm{da}=4.0$

- - Fine $d a=5.0$

- Fine $d a=6.0$

- Fine da $=6.0$

- Fine $\mathrm{da}=7.0$

- Fine da $=8.0$

- Fine $\mathrm{da}=9.0$

- Fine $\mathrm{da}=10.0$

- Fine $d_{a}=11.0$

Figure 20: Crack profiles comparison using coarse and fine mesh models.
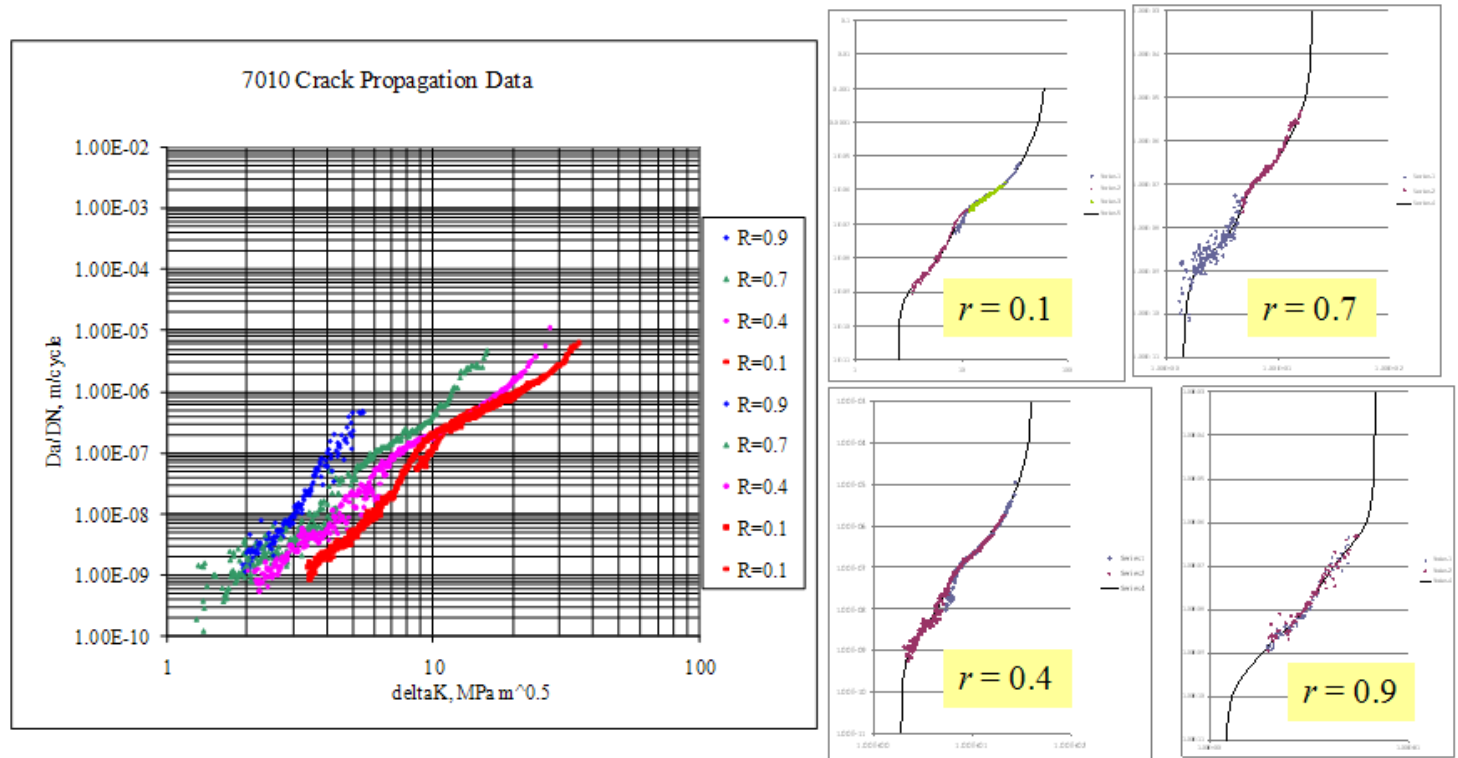

Figure 21: Load ratio dependent fatigue model at several distinct $r$ values. 